\title{
A liver stress-endocrine nexus promotes metabolic integrity during dietary protein dilution
}

\author{
Adriano Maida, ${ }^{1}$ Annika Zota, ${ }^{1}$ Kim A. Sjøberg, ${ }^{2}$ Jonas Schumacher, ${ }^{3}$ Tjeerd P. Sijmonsma, ${ }^{3,4}$ Anja Pfenninger, ${ }^{5}$ \\ Marie M. Christensen, ${ }^{2}$ Thomas Gantert, ${ }^{3}$ Jessica Fuhrmeister, ${ }^{3}$ Ulrike Rothermel, ${ }^{6}$ Dieter Schmoll, ${ }^{5}$ Mathias Heikenwälder, \\ Juan L. Iovanna, ${ }^{7}$ Kerstin Stemmer, ${ }^{8}$ Bente Kiens, ${ }^{2}$ Stephan Herzig, ${ }^{1}$ and Adam J. Rose ${ }^{3}$ \\ IInstitute for Diabetes and Cancer (IDC), Helmholtz Center Munich, and Joint Heidelberg-IDC Translational Diabetes Program, Munich, Cermany. ${ }^{2}$ Section of Molecular Physiology, August Krogh Centre, \\ Department of Nutrition, Exercise and Sports, Faculty of Science, University of Copenhagen, Copenhagen, Denmark. ${ }^{3}$ Joint Research Division Molecular Metabolic Control, German Cancer Research Center, \\ Center for Molecular Biology, Heidelberg University and Heidelberg University Hospital, Heidelberg, Germany. ${ }^{4}$ Division of Chronic Inflammation and Cancer, German Cancer Research Center, Heidelberg, \\ Germany. ${ }^{5}$ Sanofi-Aventis Deutschland GmbH, Industriepark Hoechst, Frankfurt, Germany. ${ }^{6}$ Division of Cellular and Molecular Pathology, German Cancer Research Center, Heidelberg, Germany. \\ 7Centre de Recherche en Cancérologie de Marseille, INSERM U1068, CNRS UMR 7258, Aix-Marseille Université and Institut Paoli-Calmettes, Parc Scientifique et Technologique de Luminy, \\ Marseille, France. ${ }^{8}$ Division of Metabolism and Cancer, Institute for Diabetes and Obesity, Helmholtz Centre Munich, Munich, Germany.
}

\begin{abstract}
Dietary protein intake is linked to an increased incidence of type 2 diabetes (T2D). Although dietary protein dilution (DPD) can slow the progression of some aging-related disorders, whether this strategy affects the development and risk for obesityassociated metabolic disease such as T2D is unclear. Here, we determined that DPD in mice and humans increases serum markers of metabolic health. In lean mice, DPD promoted metabolic inefficiency by increasing carbohydrate and fat oxidation. In nutritional and polygenic murine models of obesity, DPD prevented and curtailed the development of impaired glucose homeostasis independently of obesity and food intake. DPD-mediated metabolic inefficiency and improvement of glucose homeostasis were independent of uncoupling protein 1 (UCP1), but required expression of liver-derived fibroblast growth factor 21 (FGF21) in both lean and obese mice. FGF21 expression and secretion as well as the associated metabolic remodeling induced by DPD also required induction of liver-integrated stress response-driven nuclear protein 1 (NUPR1). Insufficiency of select nonessential amino acids (NEAAs) was necessary and adequate for NUPR1 and subsequent FGF21 induction and secretion in hepatocytes in vitro and in vivo. Taken together, these data indicate that DPD promotes improved glucose homeostasis through an NEAA insufficiency-induced liver NUPR1/FCF21 axis.
\end{abstract}

\section{Introduction}

The incidence of obesity is at an epidemic level worldwide. According to estimates by the World Health Organization, worldwide there are more than 1 billion overweight adults, at least 300 million of which are obese (1). Obesity is a strong risk factor for a number of diseases including type 2 diabetes (T2D), cardiovascular disease, and metabolic syndrome, and thus imposes a tremendous burden on quality of life and health care systems worldwide. Thus, there is a desperate need for more effective strategies to curtail this trend, whether through prescription of behavioral or pharmacological treatments.

Along with genetic and epigenetic predisposition, significant contributors to the escalation in obesity in recent decades are environmental factors such as diet and physical inactivity (2). Concerning diet, of the major macronutrients consumed, focus has been primarily placed on the quality and quantity of fats and carbohydrates (3), whereas the role of dietary protein has, until recently, been largely neglected (4). While it is well known that dietary protein is essential for normal growth and

Conflict of interest: A. Pfenninger and D. Schmoll are employees of Sanofi-Aventis Deutschland, a pharmaceutical company.

Submitted: December 8, 2015; Accepted: July 7, 2016.

Reference information: / Clin Invest. 2016;126(9):3263-3278. doi:10.1172/JCI85946. development, less is known about the interaction of dietary protein with systemic energy homeostasis and glucose homeostasis in adults. Indeed, the specific role of dietary protein in obesity and diabetes is less well understood and controversial (5). On one hand, the satiating effects of dietary protein are well established (6), and there are several studies in rodents $(7,8)$ and even humans (9) demonstrating higher energy intake and subsequent fat mass gain with consumption of a protein-diluted diet. The phenomenon of animals modifying their caloric intake in order to meet a daily protein target was coined the "protein leverage" hypothesis by Simpson and Raubenheimer (10). Thus, animals will hyperconsume a protein-diluted diet in order to meet this hypothetical threshold. This has led to the postulation that a societal shift towards the consumption of protein-diluted diets in the last 40-50 years may be a causal factor for heightened caloric intake and obesity (10).

On the other hand, a comprehensive diet study of mice using different ratios of the 3 major macronutrients revealed that dietary protein was the most potent macronutrient in modulating metabolic health, with worsened outcomes associating with dietary protein enrichment (11). Accordingly, intervention studies in rodents and humans have shown that an increased systemic supply of protein or amino acids (AAs) negatively affects systemic insulin action (12-14), and epidemiological studies show 

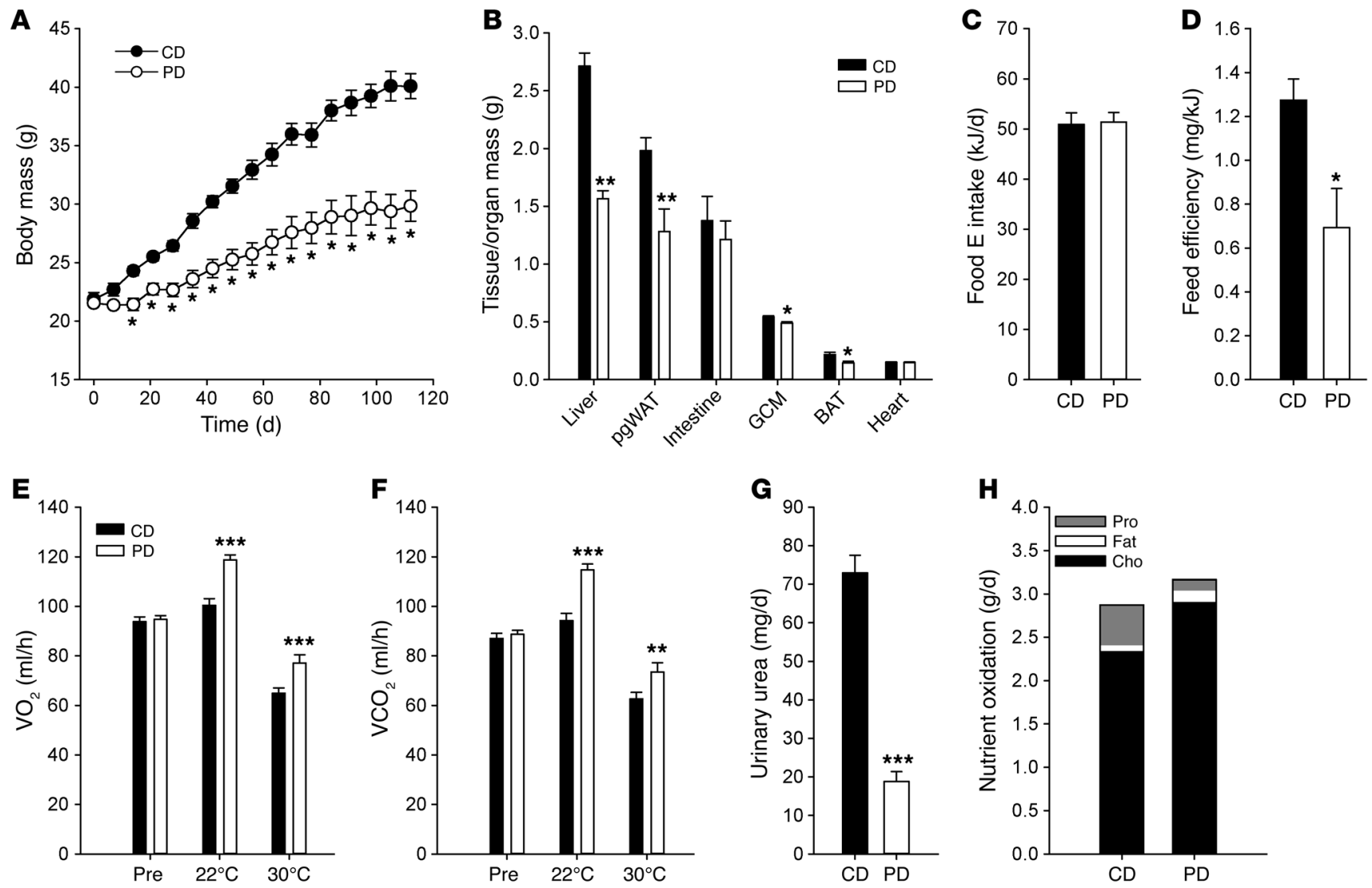

Figure 1. Chronic dietary protein dilution promotes feed inefficiency, leanness, and heightened energy expenditure. (A) Body mass accrual over 16 weeks in mice fed a control diet (CD) containing $20 \%$ caloric energy from protein (CD) or a protein-diluted (PD) diet containing $5 \%$ caloric energy from protein, diluted by added carbohydrate. $n=5$ or 6/group. (B) Tissue/organ mass at necropsy at 16 weeks in mice from A. pgWAT, perigonadal white adipose tissue; GCM, gastrocnemius complex muscle; BAT, brown adipose tissue. (C) Food energy (E) intake from individually housed mice during days 77-105 by mice from A. (D) Feed efficiency in individually housed mice from $\mathbf{A}$ during days 77-105. (E) 24-hour averaged oxygen consumption rate $\left(\mathrm{VO}_{2}\right)$ in mice fed $\mathrm{CD}$ or PD before (pre) and during 3 weeks at an ambient temperature of $22^{\circ} \mathrm{C}$ and a subsequent 2 weeks at $30^{\circ} \mathrm{C}$. Values are mean rates over a 3-day period at the end of each period when values were stable. $n=10$ /group. (F) 24-hour-averaged $\mathrm{CO}_{2}$ production rate $\left(\mathrm{VCO}_{2}\right)$ from mice as in $\mathbf{E}$. (G) Daily urinary urea production rate $\left(22^{\circ} \mathrm{C}\right)$ from mice as in $\mathbf{E}$. (H) Nutrient oxidation rates $\left(22^{\circ} \mathrm{C}\right)$ calculated from indirect calorimetry values as well as urea production. Pro, protein; Cho, carbohydrate. Data are the mean \pm SEM. ${ }^{*} P<0.05$, ${ }^{*} P<0.01$, and ${ }^{* *} P<0.001$ by 2-way repeated measures ANOVA with Holm-Sidak posthoc test (A, E, and $\mathbf{F})$, unpaired $t$ test (B-D and $\mathbf{G})$, or 1-way ANOVA with Holm-Sidak post-hoc test $(\mathbf{H})$ for significant differences between CD and PD. See also Supplemental Figure 1.

that dietary protein intake and T2D incidence are positively correlated in humans (15).

Interestingly, it was recently shown that dietary protein dilution promotes systemic energy expenditure through heightened levels of the hormone fibroblast growth factor 21 (FGF21) (16). Hence, even though dietary protein dilution may promote heightened food intake, metabolic health may pivot upon other traits, such as metabolic efficiency (17), which is the propensity of an organism to convert ingested nutrients into biomass (18), principally determined by the balance between food energy intake and somatic energy expenditure.

Here we conducted studies to comprehensively characterize the effects of dietary protein dilution (DPD) on systemic metabolism, the application of DPD in obesity-driven T2D, as well as the molecular mechanisms involved, and demonstrate that DPD promotes improved metabolic health in mice and men, at least in part through a select nonessential AA (NEAA) insufficiency-induced liver nuclear protein 1-FGF21 axis.

\section{Results}

Chronic DPD promotes metabolic inefficiency and leanness through heightened energy expenditure. To initially investigate the effects of DPD, we characterized the chronic effects of feeding a proteindiluted diet (Supplemental Table 1; supplemental material available online with this article; doi:10.1172/JCI85946DS1) to mice. DPD caused a slower body mass accrual (Figure 1A) culminating in an $~ 50 \%$ lower gain in body mass over the study period (Supplemental Figure 1A), despite only a mild effect on body length (Supplemental Figure 1B). In particular, when body composition was assessed by MRI (Supplemental Figure 1C) or by organ or tissue mass (Figure 1B), DPD reduced the mass of lean tissues such as liver and skeletal muscle, but even more striking was the effect on fat tissues, which was reflected both by lower fat gain over the study period (Supplemental Figure 1A) as well as lower fat depot tissue masses (Figure 1B). Although we observed a slight reduction in skeletal muscle mass with DPD, forelimb grip strength remained unchanged (Supplemental Figure 1D). Absolute caloric intake, as 
assessed in individually housed animals during the final 4 weeks of the experiment, did not differ between diet groups (Figure 1C), although there was a heightened intake with DPD when expressed relative to total or lean body mass (data not shown). Finally, calculation of feed efficiency, which reflects the ratio of body mass accrual to energy intake, revealed a striking $~ 40 \%$ lower feed efficiency with DPD (Figure 1D).

Given the striking effect of DPD on feeding efficiency, we next used indirect calorimetry to assess how whole-body energy metabolism was influenced by a protein-diluted diet, and whether these effects were influenced by ambient environmental temperature. In this study, body mass accrual rate was not affected until the end of the study (Supplemental Figure 1E), despite transiently enhanced food energy intake on the protein-diluted diet (Supplemental Figure $1 \mathrm{~F}$ ), again reflecting a feed inefficiency. The metabolic inefficiency caused by DPD was not due to an influence on fecal energy excretion (Supplemental Figure $1 G)$, but was rather caused by a heightened energy expenditure as reflected by increased oxygen consumption rates throughout the light-dark cycle (Figure 1E and Supplemental Figure 1, H and I), independently of changes in physical activity (Supplemental Figure 1J) and ambient housing temperature. The DPD-driven increase in $\mathrm{VO}_{2}$ was roughly paralleled by increased $\mathrm{CO}_{2}$ production rates (Figure $1 \mathrm{~F}$ and Supplemental Figure 1K), thereby resulting in only minor effects on respiratory exchange ratio (Supplemental Figure 1L). Urinary urea production was decreased by $\sim 75 \%$ (Figure $1 \mathrm{G}$ ), and as this essentially reflects systemic protein oxidation rate (19), we could calculate total and individual nutrient oxidation rates (Figure 1H), and found that DPD caused $25 \%$ and $75 \%$ higher carbohydrate and fat oxidation rates, respectively, with a $75 \%$ lower protein oxidation rate.

As increased energy expenditure is a recognized means of promoting metabolic health (17), we then assessed biomarkers of improved health such as blood serum metabolite and hormone levels (Figure 2A and Supplemental Figure 2A). There were clear effects of DPD on serum urea cycle function as assessed by reduced urea and ornithine, as well as reduced levels of certain AAs such as branched-chain AAs, and markers of protein or collagen turnover such as hydroxyproline, 1-methylhistidine, and 3-methylhistidine. However, perhaps surprisingly, there were also effects on serum lipid levels with both lower cholesterol and triglyceride, with no differences in nonesterified fatty acid (NEFA) or ketone body (i.e., $\beta$-hydroxybutyrate) levels. In terms of metabolic hormones, DPD promoted a positive hormonal profile with lower insulin (Figure 2B), insulin-like growth factor-1 (IGF1) (Figure 2C), and leptin (Figure 2D), and with higher thyroxine (Figure $2 \mathrm{E}$ ), adiponectin (Figure $2 \mathrm{~F}$ and Supplemental Figure 2, B and C), and FGF21 (Figure 2G). One key measure of metabolic health is improved glucose homeostasis, and so we assessed this in shortterm dietary protein-diluted mice. While DPD did not drastically affect the glucose excursion (Figure $2 \mathrm{H}$ ) during a glucose tolerance test, insulin levels were drastically reduced (Figure 2I), and thus insulin action was clearly improved, as indicated by a higher insulin-action index (data not shown). We further tested this by insulin tolerance testing, and DPD mice had enhanced systemic glucose lowering (Figure 2J) despite lower insulin levels (Figure $2 \mathrm{~K}$ ), again demonstrating heightened insulin action (Figure $2 \mathrm{~L}$ ), which was caused by enhanced glucose disposal in tissues such as brown and inguinal white adipose and perhaps heart (Figure $2 \mathrm{M}$ and Supplemental Figure 2D). Furthermore, DPD mice had a reduced hyperglycemic response to intraperitoneal pyruvate challenge (Supplemental Figure 2, E and F), indicating that alterations in both glucose production and clearance lead to improvements in glucose homeostasis with DPD.

Chronic DPD improves metabolic health in obesity independently of fatness. Given the enhanced glucose homeostasis in DPD, we then wanted to test the possible therapeutic potential of such a dietary strategy in mouse models of obesity-driven insulin resistance and T2D. Therefore, we employed nutritional (Supplemental Table 1) and polygenic experimental mouse models: $\mathrm{C} 57 \mathrm{Bl} / 6 \mathrm{~N}$ mice fed a high-fat diet (HFD) and the New Zealand obese (NZO) mouse strain, respectively. Similarly to the initial study (Figure 1A), lean mice of both strains fed the high carbohydrate protein-diluted diet gained less body mass (Figure 3, A and C, and Supplemental Figure 3, A and B) and exhibited only minor effects on body length (Supplemental Figure 3, C and D). However, in both HFD-induced and hyperphagic obesity, DPD did not retard weight gain (Figure 3, A and C, and Supplemental Figure 3, $\mathrm{A}$ and B) and actually increased fat depot masses (Supplemental Figure 3, E and F). On the other hand, liver mass was consistently lower in DPD mice (Supplemental Figure 3, E and F), irrespective of dietary fat or mouse strain. Nevertheless, although nutritional and polygenic obesogenic mouse models demonstrated higher feed efficiency relative to their lean counterparts with standard protein feeding, DPD consistently retarded feed efficiency (Figure 3, B and D), owing to increases in food energy intake (Supplemental Figure 3, G and $\mathrm{H}$ ).

As pronounced enhancement of carbohydrate oxidation and glucose metabolism was observed following DPD in lean mice (Figure 1), we also assessed glucose homeostasis in experimental obesity. Of note, obese DPD mice had markedly improved glucose homeostasis (Figure 3, E and F) and insulin action as assessed by the insulin sensitivity index (ISI) from fasting glucose and insulin values (Figure 3, G and $\mathrm{H}$ and Supplemental Figure 3, I and J) or an ISI calculated from glucose and insulin areas under the curves (Supplemental Figure 3, I-L), and there was a close positive relationship between these 2 indices (Supplemental Figure $3 \mathrm{M}$ ). Strikingly, the DPD NZO mice did not develop overt glucosuria (Figure 3I), indicating a complete prevention of T2D. In a separate study, we also assessed tissue-specific glucose disposal (Figure 3J), and could observe increased rates of glucose uptake particularly in the heart $(\sim 5$-fold) and brown adipose tissue (BAT) $(\sim 1.5$-fold $)$ in DPD NZO mice.

Since the former 2 models addressed whether DPD could prevent the development of obesity-driven insulin resistance (IR) or T2D, we then wanted to test whether DPD could serve to reverse existing obesity-driven IR. As such, we subjected $\mathrm{C} 57 \mathrm{Bl} / 6 \mathrm{~N}$ mice to either high-carbohydrate or high-fat diets for 8 weeks prior to switching part of the groups to respective protein-diluted diets. The effects of DPD on body mass accrual and composition (Supplemental Figure 3, N-Q) were consistent with our prior observations (Figure 3 and Supplemental Figure 3). Of note, however, was that the effects of DPD could reverse the effects of preexisting insulin resistance (Figure $3 \mathrm{~K}$ ), based 
A

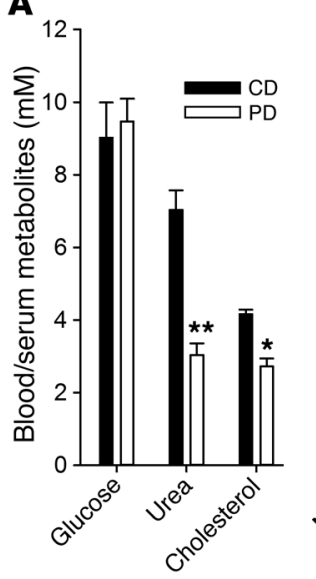

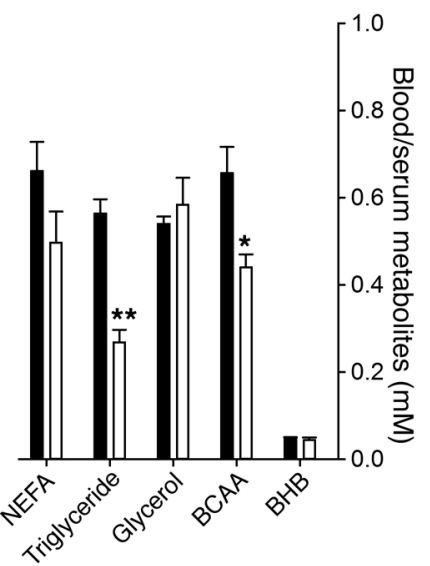

B

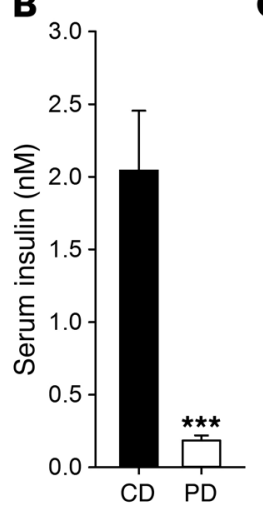

C

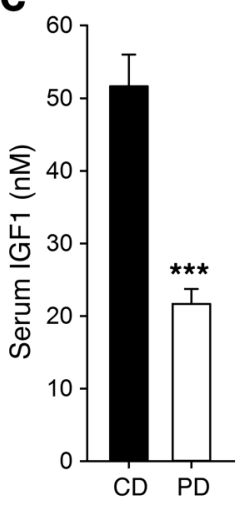

D

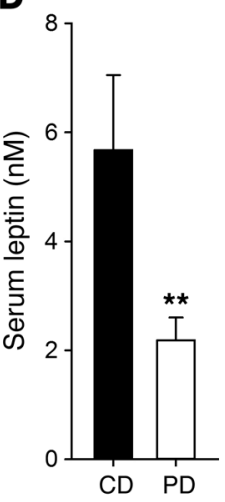

E

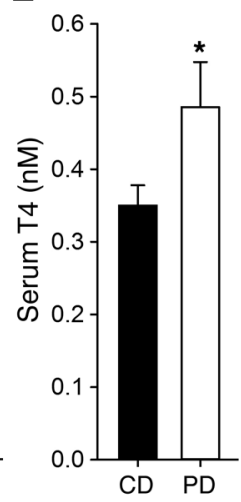

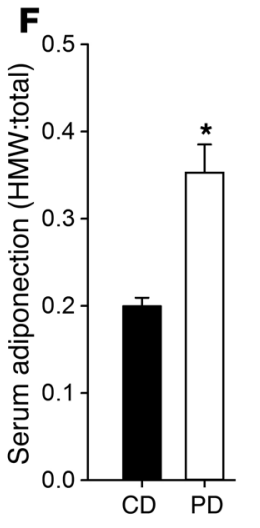

G
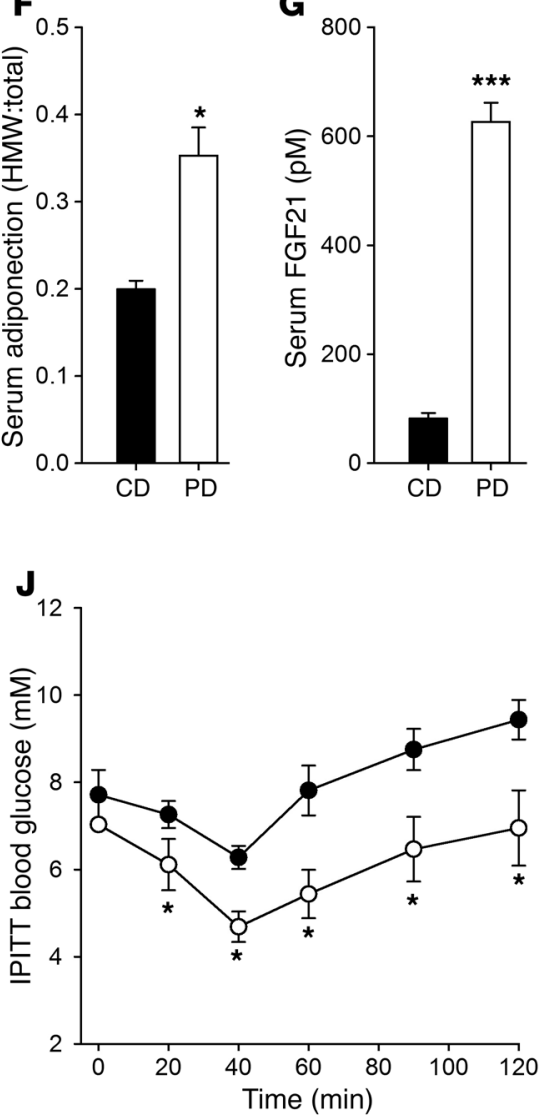

H

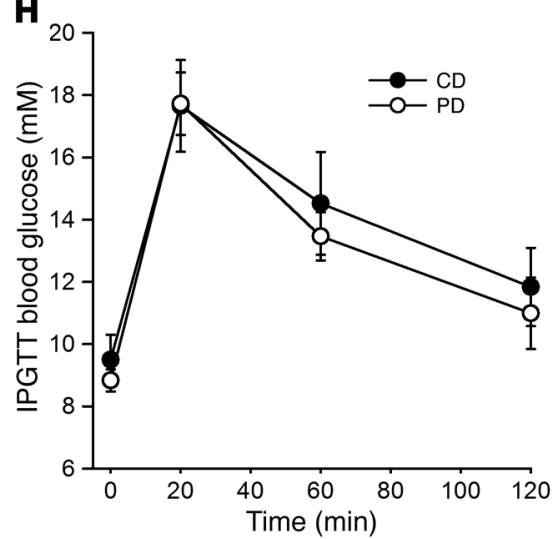

K

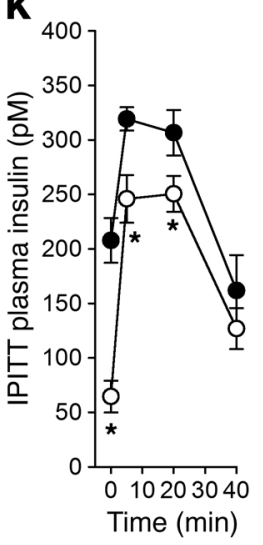

L

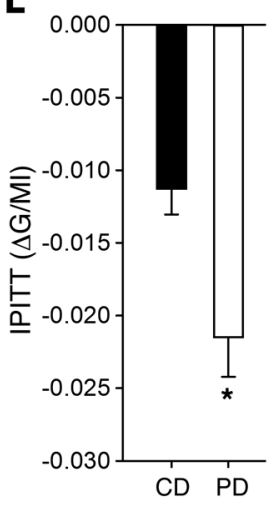

I

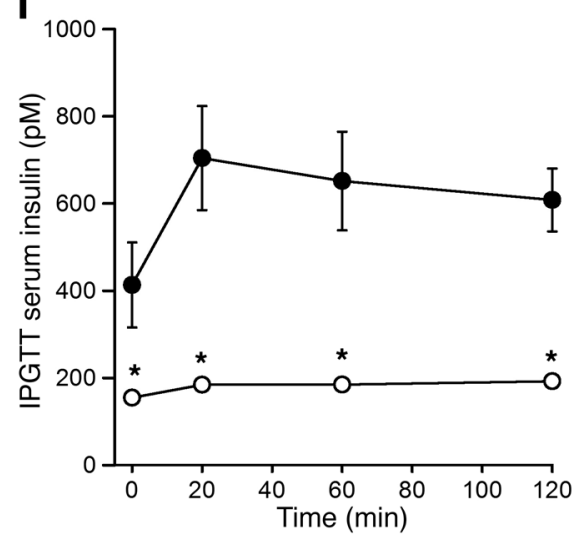

M

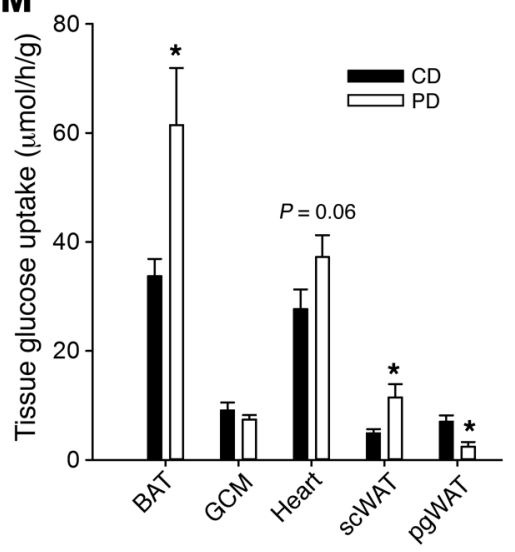

Figure 2. Chronic dietary protein dilution promotes a metabolite and hormonal profile reflective of improved metabolic health as well as enhanced glucose metabolism. (A) Blood and serum metabolites from mice as in Figure 1A. NEFA, nonesterified fatty acids; BCAA, branched-chain amino acids; BHB, $\beta$-hydroxybutyrate. (B) Serum insulin levels in mice from Figure 1A. (C) Serum insulin-like growth factor 1 (IGF1) levels in mice from Figure 1A. (D) Serum leptin levels in mice from Figure 1A. (E) Serum thyroxine (T4) levels in mice from Figure 1A. (F) Serum adiponectin levels in mice from Figure 1A. (C) Serum fibroblast growth factor 21 (FGF21) levels in mice from Figure 1A. (H) Blood glucose excursion during an intraperitoneal glucose tolerance test (IPGTT) in mice fed a control diet (CD) containing 20\% caloric energy from protein (CD) or a protein-diluted (PD) diet containing $5 \%$ caloric energy from protein, diluted by added carbohydrate, for 2 weeks. $n=5$ or 6/group. (I) Serum insulin excursion in mice treated as in H. (J) Blood glucose excursion during an intraperitoneal insulin tolerance test (IPITT) in mice treated as in $\mathbf{H}$. $n=5$ or $6 /$ group. (K) Serum insulin excursion in mice treated as in J. (L) Insulin action index as represented by the maximal change in blood glucose $(\Delta \mathrm{C}$ ) divided by the maximal level of serum insulin (MI) from mice treated as in H. (M) Tissue glucose uptake rates of mice treated as in J. pgWAT, perigonadal white adipose tissue; scWAT, subcutaneous white adipose tissue; GCM, gastrocnemius complex muscle; BAT, brown adipose tissue. $n=6$ /group. Data are the mean $\pm \mathrm{SEM}$. ${ }^{*} P<0.05$, ${ }^{* *} P<0.01$, and ${ }^{* * *} P<0.001$ by unpaired $t$ test (A-G, L, and $\mathbf{M}$ ) or 2-way repeated measures ANOVA with Holm-Sidak post-hoc test (H-K) for significant differences between CD and PD. See also Supplemental Figure 2. 

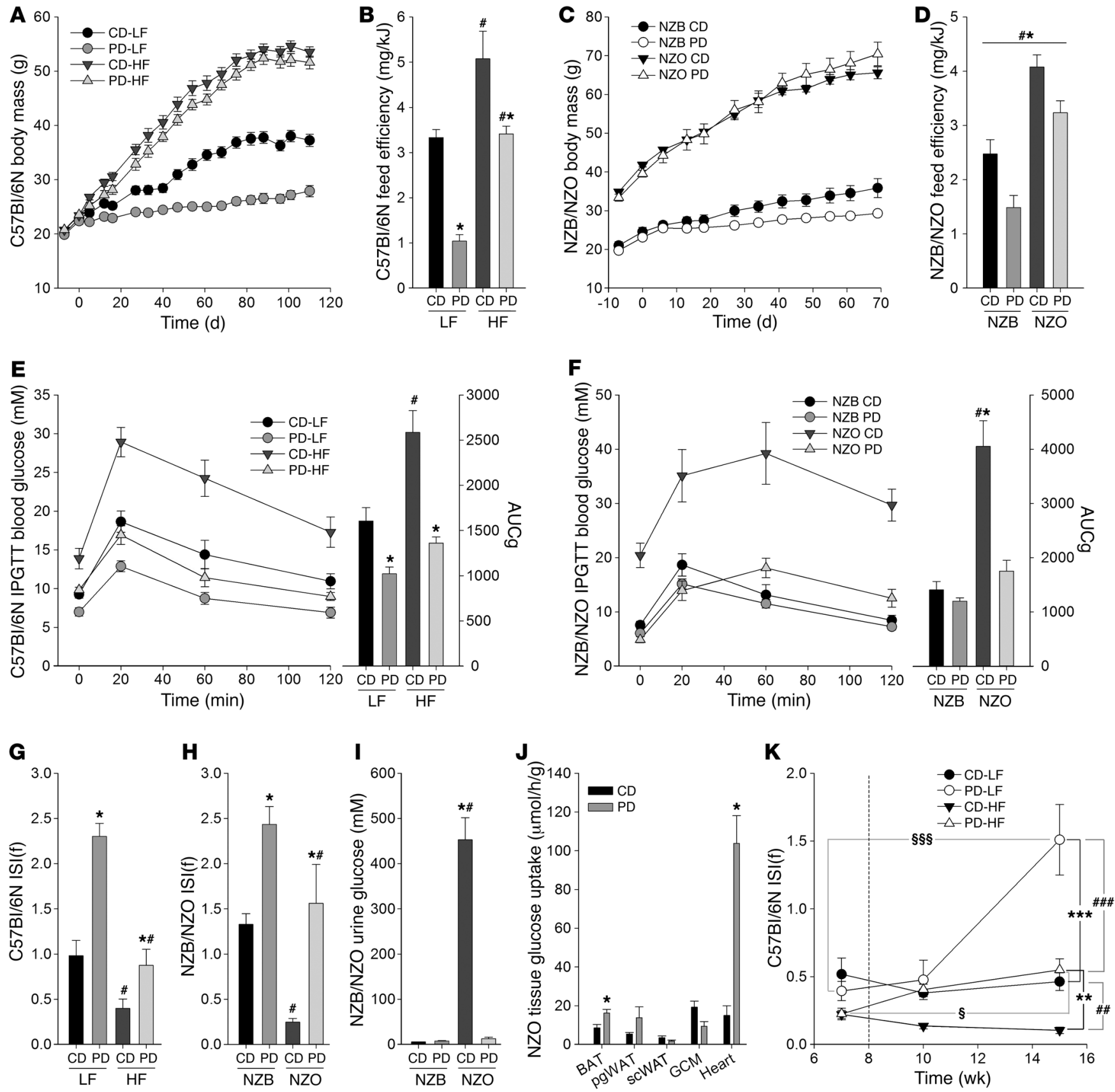

Figure 3. Dietary protein dilution promotes improved glucose homeostasis independently of obesity. (A) Body mass accrual of C57BI/6N mice fed either control diet (CD) containing $20 \%$ caloric energy from protein or a protein-diluted (PD) diet containing $5 \%$ caloric energy from protein, diluted by added carbohydrate, with either $10 \%$ (LF) or $60 \%$ (HF) calories from fat. $n=6-8$ /group. (B) Feed efficiency from mice treated as in A. (C) Body mass accrual in New Zealand Black (NZB) or New Zealand Obese (NZO) mice fed a CD containing $20 \%$ caloric energy from protein or a PD containing $5 \%$ caloric energy from protein, diluted by added carbohydrate. $n=6$-8/group. (D) Feed efficiency from mice treated as in C. (E) Blood glucose excursion during an intraperitoneal glucose tolerance test (IPGTT) in mice from A. AUCg, glucose area under the curve. (F) Blood glucose excursion during an IPGTT in mice from C. (G) Fasting insulin sensitivity index [ISI(f)] from mice as in $\mathbf{A}$. (H) ISI(f) from mice treated as in C. (I) Urinary glucose levels from mice treated as in $\mathbf{C}$. (J) Tissue glucose uptake rates during an IPGTT from mice treated as in C. pgWAT, perigonadal white adipose tissue; scWAT, subcutaneous white adipose tissue; GCM, gastrocnemius complex muscle; BAT, brown adipose tissue. $n=5$ or 6/group. (K) ISI(f) was measured from mice at selected time points before and after maintenance on the same diet or a switch (day 56, indicated by a vertical dashed line) to a respective protein-diluted diet. $n=7$ or $8 /$ group. Data are the

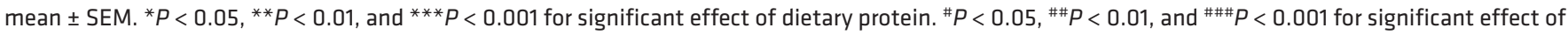
dietary fat/strain. ${ }^{\S} P<0.05$ and ${ }^{\$ \S} P<0.001$ for significant effect of time. Statistical tests used were 2-way ANOVA with Holm-Sidak post-hoc test (B, D-I), unpaired $t$ test (J), and 2-way repeated measures ANOVA with Holm-Sidak post-hoc test (K). See also Supplemental Figure 3. 
A

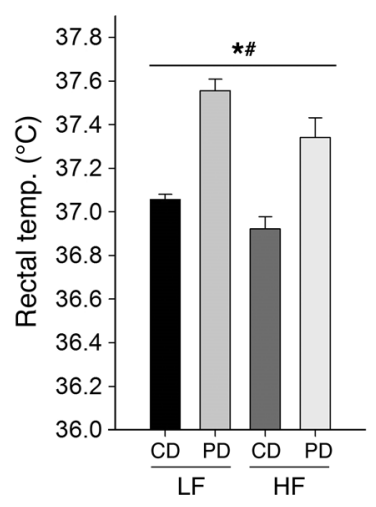

D

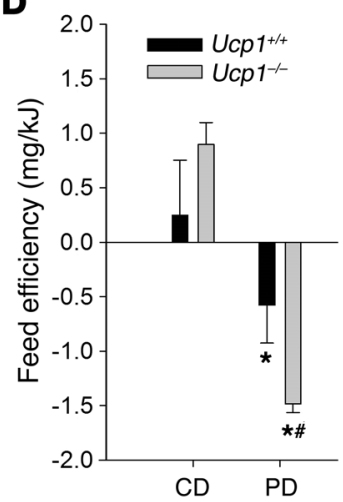

B

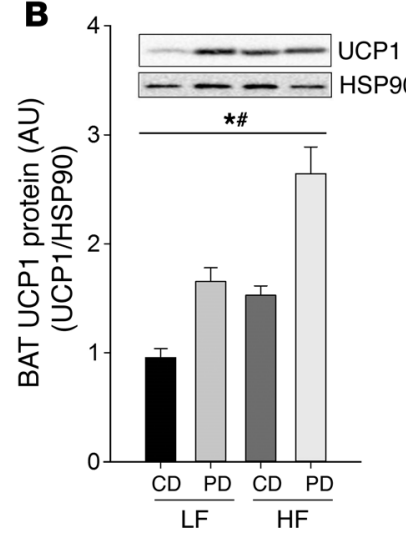

E

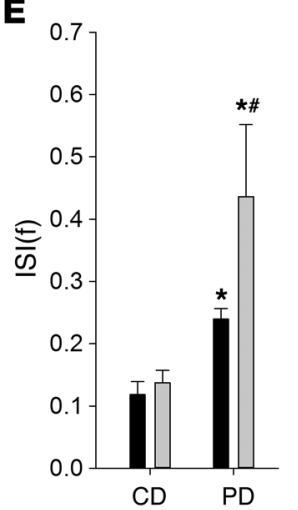

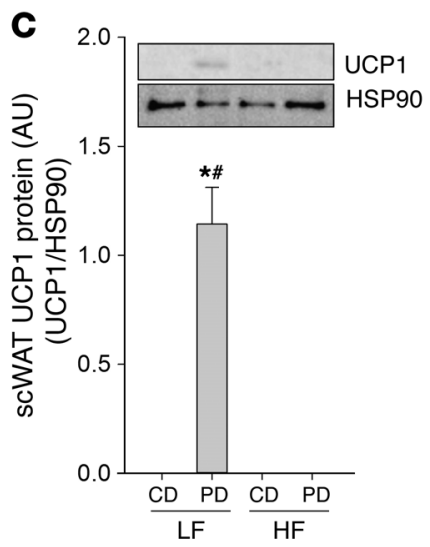

$\mathbf{F}$

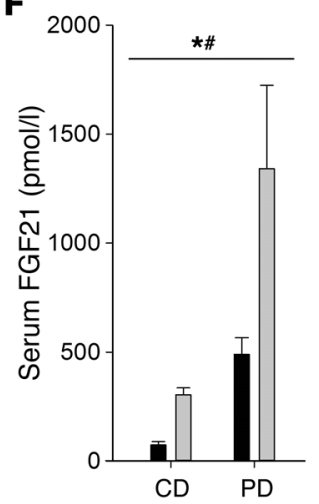

Figure 4. Dietary protein dilution promotes metabolic inefficiency and improves glucose homeostasis independently of uncoupling protein 1 (UCP1) expression. (A) Rectal temperature in mice treated as in Figure $3 \mathrm{~A}$. (B) Brown adipose tissue (BAT) UCP1 protein expression in mice treated as in Figure 3A. (C) Subcutaneous white adipose tissue (scWAT) UCP1 protein expression in mice treated as in Figure 3A. (D) Feed efficiency of wild-type $\left(U c p 1^{1^{+/}}\right)$and Ucp1 knockout (Ucp1 $\left.1^{-/-}\right)$ mice fed a control diet (CD) containing $20 \%$ caloric energy from protein or a protein-diluted (PD) diet containing 5\% caloric energy from protein, diluted by added carbohydrate. Studies were conducted for 6 weeks. $n=6-9 / g r o u p$. (E) Fasting insulin sensitivity index [ISI(f)] from mice treated as in D. (F) Serum fibroblast growth factor 21 (FGF21) levels in mice treated as in $\mathbf{D}$. Data are the mean \pm SEM. ${ }^{*} P<0.05$ for significant effect of dietary protein; ${ }^{\#} P<0.05$ for significant effect of dietary fat or genotype, 2-way ANOVA with Holm-Sidak post-hoc test. See also Supplemental Figure 4 upon fasting blood glucose (Supplemental Figure 3R) and serum insulin (Supplemental Figure 3S) analyses.

DPD effects are independent of UCP1 function. The effects of DPD on feed efficiency (Figure 3) were also reflected by a higher core body temperature (Figure $4 \mathrm{~A}$ ), indicative of diet-induced thermogenesis. Given that UCP1 is considered to be the only protein capable of conferring nonshivering thermogenesis in mammals (20) and that we observed changes in Ucp1 mRNA in BAT and white adipose tissue (WAT) from DPD mice (data not shown), we then assessed whether these changes were recapitulated at the level of UCP1 protein expression. Indeed, both BAT (Figure 4B) and subcutaneous WAT (scWAT) (Figure 4C), UCP1 expression essentially reflected that observed in mRNA expression levels. Initially we assessed the involvement of sympathetic thermogenic mechanisms by conducting a diet-switch study in mice adapted to thermoneutral conditions, resulting in reduced sympathetic tone, thermogenic energy requirements, and UCP1 expression (21). However, DPD-induced feed inefficiency (Supplemental Figure $4 \mathrm{~A}$ ) and increased core body temperature (Supplemental Figure $4 \mathrm{~B})$ were preserved at thermoneutrality. To further test the involvement of canonical thermogenic mechanisms, we placed germline UCP1 knockout mice and wild-type littermates on normal as well as protein-diluted diets. Similarly to the thermoneutrality experiment, the effects of DPD on body composition (Supplemental Figure 4, C and D), food energy intake (Supplemental Figure 4E), and thus feed efficiency (Figure 4D) were not reversed by UCP1 loss of function, and were perhaps even exacerbated. Similarly, there was no attenuation of DPD effects on glucose metabolism in the absence of UCP1, as assessed by ISI (Figure 4E and Supplemental
Figure 4, F and G). Although it is thought that UCP1 elimination may recruit compensatory thermogenic mechanisms at standard housing temperatures (21), similar results were obtained when we conducted these $U c p 1^{-/-}$studies under thermoneutral conditions (data not shown). Lastly, DPD resulted in potentiation of serum FGF21 levels in mice with absent UCP1 (Figure 4F), indicating that the exacerbated effects of DPD could be driven by heightened FGF21 in this setting.

$D P D$ effects require liver-derived FGF21. Given that we consistently observed higher serum levels of FGF21 with DPD (Figures 2 and 4, and Supplemental Figure 5A), we then tested whether FGF21 is required for the metabolic phenotype of DPD. Initially, we subjected groups of $\mathrm{Fg} 2 \mathrm{1}^{+/+}$and germline $\mathrm{Fg} 2 \mathrm{I}^{-1^{--}}$littermate mice to a low-fat protein-diluted or control diet (CD) in a counterbalanced, crossover manner and examined systemic nutrient oxidation rates via a combination of indirect calorimetry and urinary $\mathrm{N}$ excretion. Relative to $\mathrm{CD}$, DPD enhanced $\mathrm{O}_{2}$ consumption (Supplemental Figure 5B) and $\mathrm{CO}_{2}$ production (Supplemental Figure $5 \mathrm{C}$ ), in wild-type but not Fgf21-deficient mice. DPD depressed urinary $\mathrm{N}$ loss, but this was not different between genotypes (Supplemental Figure 5D). Taken together (19), our data show that DPD enhanced carbohydrate (Figure 5A) and fat (Figure 5B) oxidation, with no differences in the suppression of protein oxidation (Figure $5 C)$, culminating in an overall enhancement of total nutrient oxidation (Figure 5D) in $\mathrm{Fg} f 21^{+/+}$but not $\mathrm{Fg} 221^{-/-}$mice.

In follow-up studies, we conducted more long-term experiments to assess glucose homeostasis. In wild-type littermates, DPD had the expected effect of augmenting serum FGF21 (Supplemental Figure 5E) and attenuating feed efficiency (Figure 5E), 

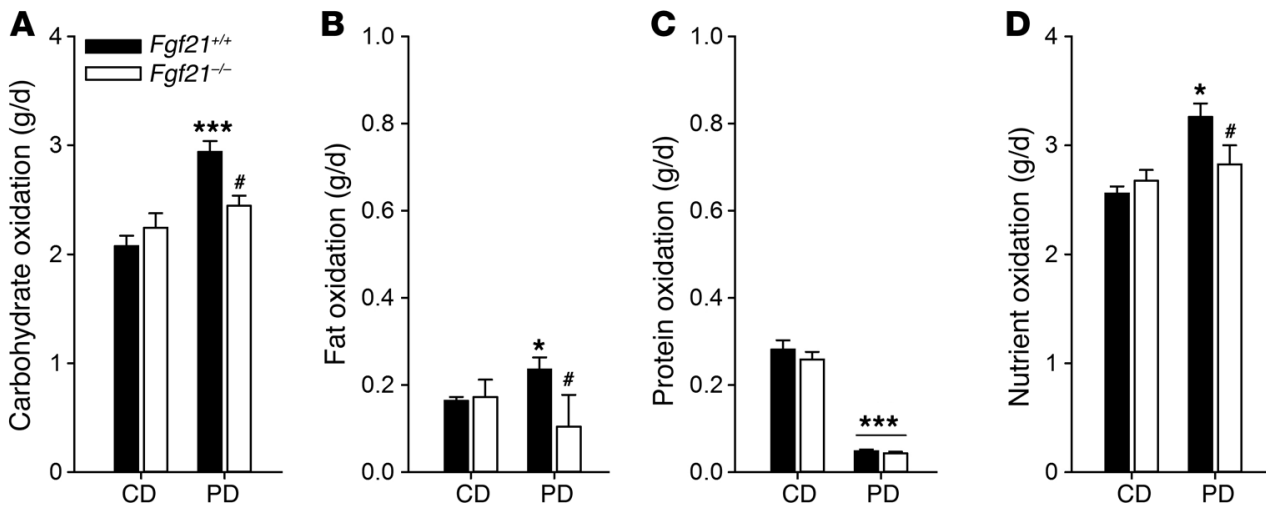

Figure 5. Liver-derived FGF21 is required for the metabolic effects of dietary protein dilution. (A) Carbohydrate oxidation rate of whole-body Fgf $21^{-/-}$and $\mathrm{Fgf2} 21^{/+}$mice studied on low-fat control diet (CD) or proteindiluted (PD) in a balanced, crossover manner. $n=8-10 /$ group. (B) Fat oxidation rate of mice treated as in $\mathbf{A}$. (C) Protein oxidation rate of mice treated as in A. (D) Total nutrient oxidation rate of mice treated as in A. (E) Feed efficiency over 4 weeks in whole-body $\mathrm{Fgf21^{-/- }}$ and Fgf $21^{+/+}$mice on low-fat CD or PD. $n=5$ or 6/group. (F) Insulin sensitivity index
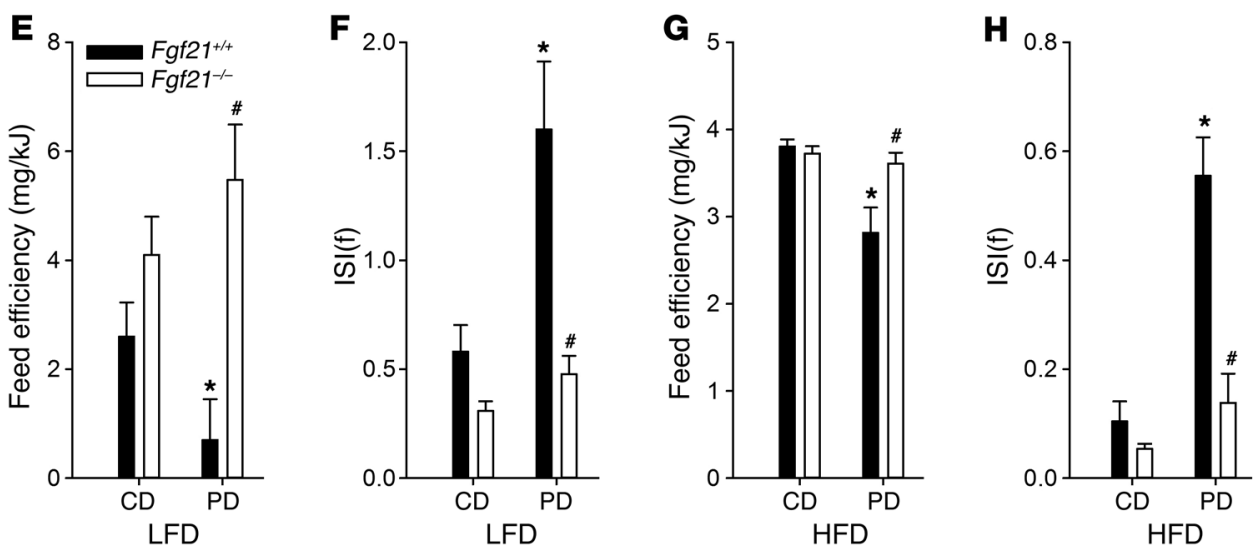
(ISI) from mice treated as in A. (C) Feed efficiency over 8 weeks in whole-body $\mathrm{Fgf21^{-/- }}$ and $\mathrm{Fgf21^{+/+ }}$ mice fed high-fat CD or PD for 16 weeks. $n=5$ or $6 /$ group. (H) ISI from mice treated as in C. (I) Tissue Fgf21 mRNA expression in C57BI/6N mice on $C D$ or $P D$ treated as in Figure $1 A$. (J) Endpoint serum FGF21 levels in CD or PD-fed C57BI/6N mice given adenoviruses overexpressing negative control (NC) or Fgf21-selective shRNAs in the liver. (K) Feed efficiency over 8 days of mice from $\mathbf{D}$. (L) ISI of mice from $\mathbf{D}$. Data are presented as the mean \pm SEM.

${ }^{*} P<0.05,{ }^{* *} P<0.001$ for differences between diets. ${ }^{\sharp} P<0.05$ for differences between genotype/virus. Statisti-
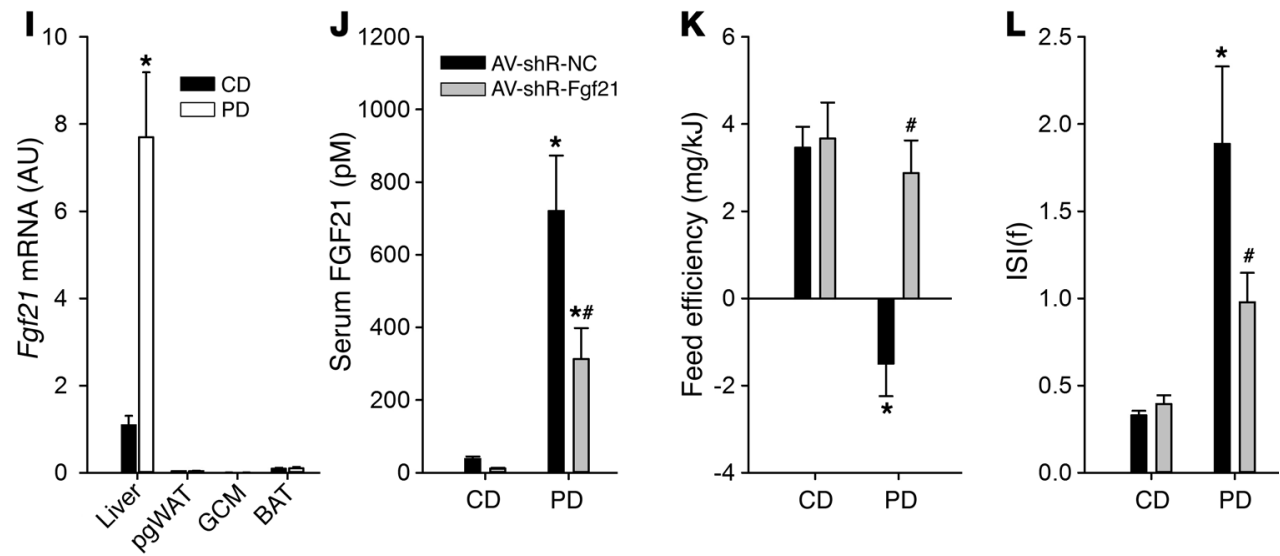
cal tests used were 2-way repeated measures ANOVA with Holm-Sidak post-hoc test (A-D), 2-way ANOVA with Holm-Sidak post-hoc test (E-L), and unpaired $t$ test (I). See also Supplemental Figure 5

owing to a slightly higher energy intake (Supplemental Figure $5 \mathrm{~F}$ ), despite lower body mass gain (Supplemental Figure 5G). In stark contrast, DPD had no effects on energy intake (Supplemental Figure 5F) or body mass gain (Supplemental Figure 5G), and thus did not influence metabolic efficiency in mice with germline deletion of Fgf21 (Figure 5E). In contrast to liver mass, which was lowered by DPD irrespective of genotype, DPD augmented fatness only in mice with abrogation of the Fgf 21 gene (Supplemental Figure 5H). In addition to metabolic efficiency, the salutary effects of DPD in heightening insulin sensitivity (Figure $5 \mathrm{~F}$ and Supplemental Figure 5, I and J) were completely dependent upon FGF21. To then test whether FGF21 was required for the effects of DPD to retard obesity-driven metabolic dysfunction, we conducted studies using high-fat protein-diluted or CD in germline $\mathrm{Fg} f 21^{-/-}$mice. Again, DPD attenuated feed efficiency in wild-type mice (Figure 5G), mostly owing to enhanced food energy intake (Supplemental Figure 5K) despite unaltered body mass accrual (Supplemental Figure 5L), effects that were abrogated in the Fgf $21^{-/}$mice. Despite no effects of DPD on body mass accrual in either genotype, there were stark differences in body composition (Supplemental Figure 5M), particularly liver and perigonadal WAT (pgWAT). In particular, whereas the low-fat PD caused an FGF21-dependent contraction of WAT, high-fat PD caused an FGF21-depdendent expansion of pgWAT. In terms of metabolic health, again PD could retard obesity-driven impaired systemic glucose homeostasis (Figure 5H), which was FGF21 dependent, owing to decreases in both fasting blood glucose (Supplemental Figure 5N) and insulin (Supplemental Figure 5O).

These results illustrated a clear dependence upon the Fgf 21 locus for the metabolic signature of DPD, and prompted us to 

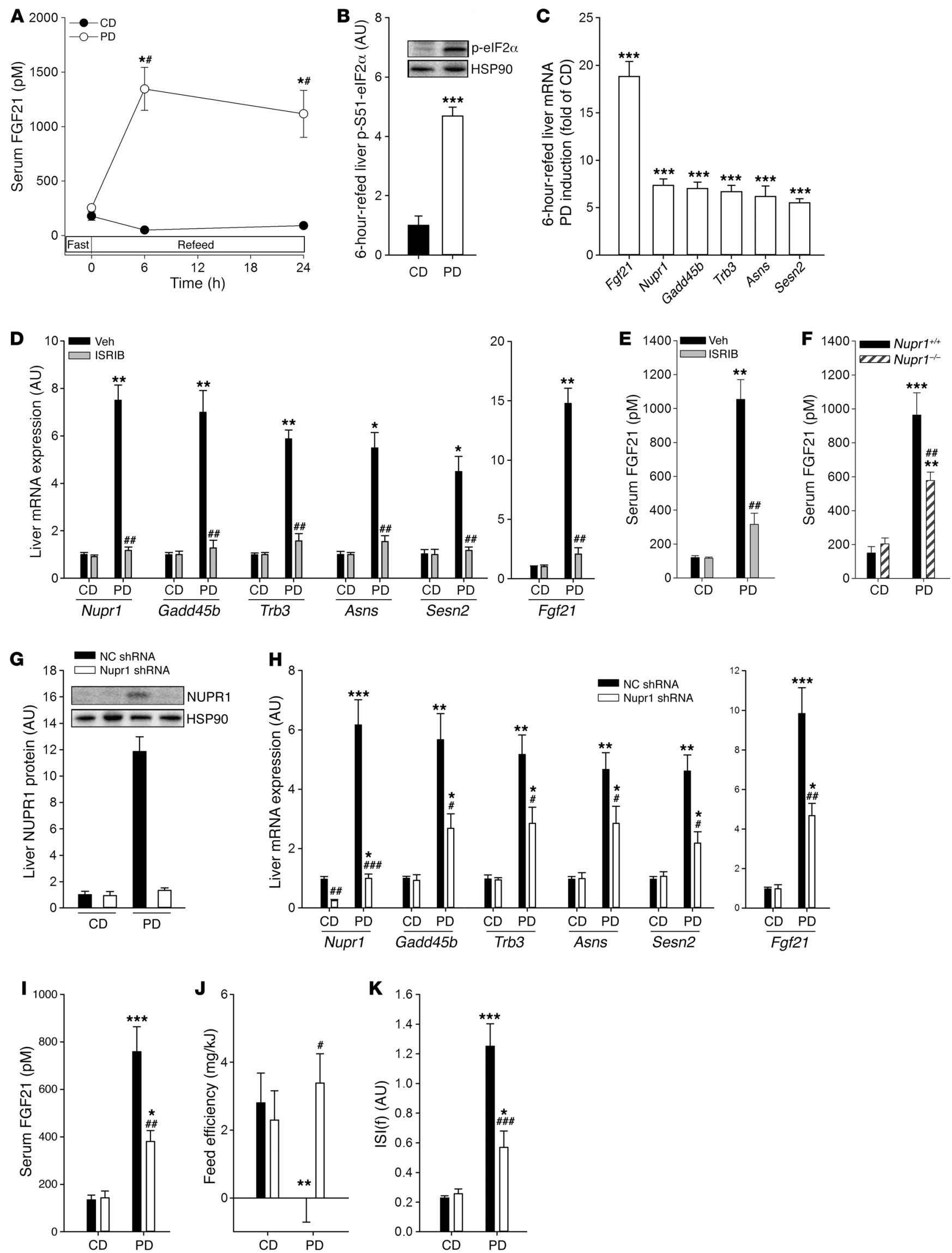
Figure 6. The integrated stress response mediates liver FGF21 induction and associated metabolic remodeling with dietary protein dilution via nuclear protein 1. (A) Serum FGF21 levels following overnight fasting and refeeding in mice on control (CD) or protein-diluted (PD) diets for 3 weeks. $n=5$ /group. (B) Liver phospho-Ser51-eukaryotic initiation factor $2 \alpha$ (p-S51elF2 $\alpha$ ) levels in overnight fasted and then 6 -hour-refed mice following a 2 -week adaptation to the respective diets. $n=6$ /group. Representative images shown are from the same samples blotted on different membranes on separate occasions. (C) Liver mRNA levels of fibroblast growth factor 21 (Fgf21), nuclear protein 1 (Nupr1), growth arrest and DNA damageinduced 45 beta (Gadd45b), tribbles homologue 3 (Trb3), asparagine synthetase (Asns), and sestrin 2 (Sesn2) of mice treated as in A. (D) Liver mRNA levels of Nupr1, Gadd45b, Trb3, Asns, Sesn2, and Fgf21 of mice adapted to CD or PD for 2 weeks, overnight fasted, and then refed and administered integrated stress response inhibitor (ISRIB) or vehicle. $n=5$ / group. (E) Serum FGF21 levels from mice treated as in D. (F) Serum FGF21

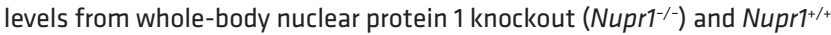
mice adapted to low-fat CD or PD and subjected to overnight fasting and refeeding. $n=5$ or 6/group. (C) Liver NUPR1 protein expression in CD- or $\mathrm{PD}$-fed mice given adenoviruses overexpressing negative control (NC) or Nupr1-selective shRNAs in the liver using a similar experimental design as in Supplemental Figure 5P. $n=5$ or 6/group. (H) Liver mRNA expression of Nupr1, Gadd45b, Trb3, Asns, and Sesn2 in mice treated as in G. (I) Serum FGF21 levels of mice treated as in G. (J) Feed efficiency of mice treated as in G. (K) Fasting insulin sensitivity index [ISI(f)] of mice treated as in $\mathbf{G}$. Data are presented as the mean $\pm \mathrm{SEM} .{ }^{*} P<0.05,{ }^{* *} P<0.01,{ }^{* * *} P<0.001$

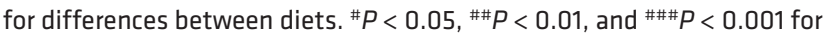
differences between genotype/virus. Statistical tests used were 2-way repeated measures ANOVA with Holm-Sidak post-hoc test (A), unpaired $t$ test (B and $\mathbf{C}$ ), and 2-way ANOVA with Holm-Sidak post-hoc test (D-K). See also Supplemental Figure 6.

examine whether higher circulating FGF21 could be ascribed to higher production by a particular tissue. A quantitative PCR (qPCR) screen of a variety of fatty and lean tissues (Figure 5I) highlighted the liver as the most likely source of higher levels of circulating FGF21 following DPD. Intestine, kidney, heart, and scWAT had relatively weak levels of Fgf21 expression, which was not modified by DPD (data not shown). To test whether liverderived FGF21 coordinates the metabolic response to DPD, we administered specific or negative control shRNA-overexpressing adenoviruses to achieve hepatic knockdown of $F g f 21$ in the liver (Supplemental Figure 5, P and Q). Liver-specific knockdown of Fgf21 resulted in correspondingly lower circulating FGF21 (Figure 5J). Recapitulating our observations with whole-body Fgf21 deletion, knockdown of hepatic $\mathrm{Fg} 221$ completely blocked the effect of DPD in reducing feed efficiency (Figure 5K), which was driven by prevention of body mass loss (Supplemental Figure 5, R and S). Moreover, the improved glucose metabolism with DPD was also dependent upon liver-derived FGF21 (Figure 5L and Supplemental Figure 5, T and U).

The integrated stress response mediates liver FGF21 induction and associated metabolic remodeling via nuclear protein 1 . To obtain insight into the mechanism by which FGF 21 might be regulated by DPD, we examined serum FGF21 levels over 24 hours in response to fasting and refeeding in mice adapted to either protein-diluted diet or CD. Following an overnight fast, levels of serum FGF21 were not significantly different between mice on the 2 diets, and $C D$ refeeding resulted in the expected decline in hormone levels over time (Figure 6A). In sharp contrast, refeeding a protein-diluted diet resulted in sharp and sustained elevation in serum FGF21 (Fig- ure 6A). Elevated serum FGF21 levels in mice on DPD for several months regressed completely within hours of switching mice back onto the CD (Supplemental Figure 6A), illustrating the dynamic regulation of this hormonal response to diluted protein intake.

As it has been reported that the integrated stress response (ISR) may be part of the mechanism by which DPD affects liver FGF21 (16), we also examined this and observed that in refed mice, there was higher phosphorylation of S51 (p-S51) in eukaryotic initiation factor $2 \alpha$ (eIF2 $\alpha$ ) (Figure 6B), a bona fide molecular readout of the ISR (22). This, along with a higher Fgf21, corresponded to an increase in certain transcripts that are known to be increased by activation of the ISR, such as Nupr1 (23,24), Gadd45b (25), Trb3 (26), Asns (25), and Sesn2 (27), thus comprising a liver gene expression signature of the ISR (GES-I) with DPD in vivo (Figure 6C and Supplemental Figure 6, B and C). Thus, we chose to examine this further and conducted an experiment to test whether activation of the ISR is required for the induction of liver Fgf 21 by DPD. As such, we treated DPD-refed mice with a small molecule known as integrated stress response inhibitor (ISRIB) (28), which acts downstream of p-S51-eIF2 $\alpha$ to retard the effects of upstream ISR signaling (29), and could observe a complete retardation of the liver GES-I with PD (Figure 6D), thereby demonstrating the effectiveness of our strategy. Similarly, liver Fgf21 mRNA (Figure 6D) and serum FGF21 (Figure 6E) levels were reduced by ISR inhibition, selectively with DPD.

Conceivably, the upregulation of $F g f 21$ transcription in the liver upon DPD could be due to enhanced transcriptional rate or mRNA stability. To assess this, we examined both the mature and immature Fgf21 transcripts within the same sample set (Supplemental Figure 6D), and both were higher to a similar extent with DPD, indicating that enhanced transcriptional rate is the main mechanism by which DPD regulates FGF21 levels. Given this information, we then sought to elucidate a mechanism by which this occurs and thus chose to examine the transcriptional regulator nuclear protein 1 (NUPR1, also known as $\mathrm{p} 8$ or COM1; ref. 30 ), as from our previous experiments (i.e., Figure 6, C and D, and Supplemental Figure 6B) we demonstrated that the expression of Nupr1 was potently upregulated in the liver with DPD, which was dependent on the ISR. As such, to establish that Nupr1 upregulation was a viable mechanism for upregulating $\mathrm{Fgf} 21$ transcription, we first demonstrated that, similarly to Nupr1 mRNA (Figure 6C), NUPR1 protein expression was higher with DPD (Supplemental Figure 6E). Also, the upregulation of Nupr1 occurs prior to the upregulation of $F g f 21$ expression in response to AA deprivation in vitro (Supplemental Figure 6, F and G), a prerequisite for potential transcriptional regulation. Encouraged by these findings, we then examined germline $\mathrm{NuprI}^{--}$mice, and when compared with corresponding $\mathrm{Nupr}^{+/+}$mice, the upregulation of serum FGF21 with DPD was less pronounced (Figure 6F). However, the metabolic readouts such as feed efficiency and insulin action were not significantly different in the germline $\mathrm{Nupr}^{-/-}$mice (data not shown), perhaps due to contrasting effects of NUPR1 in other tissues such as the pancreas (31) and heart (32). Thus, we adopted a liverrestricted strategy of Nupr1 loss of function as conducted previously for Fgf21 (Supplemental Figure 5P). Strikingly, along with NUPR1 (Figure 6G), we demonstrated that the liver GES-I with DPD was substantially dampened by NUPR1 knockdown (Figure 

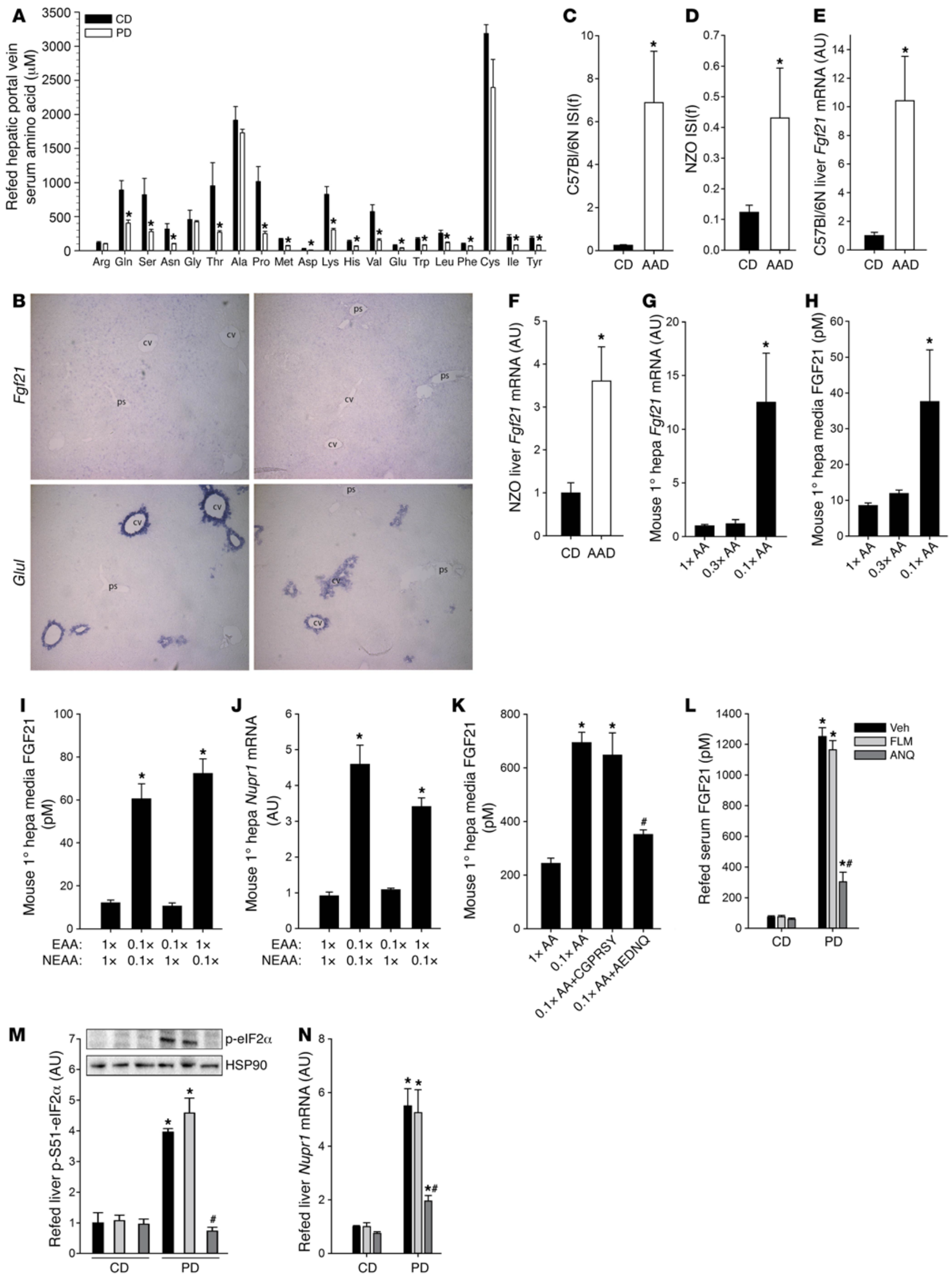
Figure 7. Nonessential amino acid dilution triggers the liver integrated stress response and FGF21 production. (A) Hepatic portal vein blood serum amino acid (AA) profile in mice adapted to and then fasted and refed control (CD) or protein-diluted (PD) diet. $n=4$ or 5/group. (B) In situ hybridization of PD-refed mouse livers serially cut and stained with probes for either Fgf21 or the central vein (cv) marker glutamine synthetase (Glul). Fgf21 transcripts surround vessels negative for Glul, suggesting portal space (ps) localization. Representative images are from liver sections taken from 2 different mice. Magnification: $\times 100$. (C) Fasting insulin sensitivity index [ISI(f)] in [57BI/6N mice fed diets with protein replaced by a casein-based AA mixture (18 kcal\% or $4.5 \mathrm{kcal} \%$ ) for 3.5 months. $n=6$ or $7 /$ group. (D) ISI(f) in NZO mice fed diets with protein replaced by a casein-based AA mixture (18 kcal\% or $4.5 \mathrm{kcal} \%$ ) for $\sim 10$ weeks. $n=5$ or 6/group. (E) Liver Fgf21 mRNA levels from mice treated as in C. (F) Liver Fgf21 mRNA levels from mice treated as in D. (G) Effect of media total AA restriction on Fgf21 mRNA in cultured primary mouse hepatocytes (hepa). $n=4$ /group. (H) Media FGF21 levels from hepatocytes treated as in G. (I) Effect of media total AA restriction, with or without differential essential (EAA) or nonessential (NEAA) amino acid supplementations on media FGF21 levels in cultured primary mouse hepatocytes. $n=4 /$ group. (J) Nupr1 mRNA levels from hepatocytes treated as in I. (K) Select NEAAs ( $A, E, D, N$, and $Q$ ), but not others ( $, ~ G, P, R, S$, and $Y$ ) attenuate media FGF21 induction by AA restriction in cultured primary mouse hepatocytes. The indicated AAs were added to match their concentration in the $1 \times A A$ group. $n=4$ /group. (L) Serum FGF21 levels following overnight fasting and refeeding in mice adapted for 2 weeks to $C D$ or PD combined with intraperitoneal administration of vehicle $(0.9 \% \mathrm{NaCl})$, select EAA (i.e., Phe, Leu, Met [FLM]), or NEAA (i.e., Ala, Asn, Gln [ANQ]) (8 mg each, 24 mg; 1 mg/g body mass). $n=4$ /group. (M) Liver phospho-Ser51-eukaryotic elongation factor $2 \alpha$ (p-S51-elF $2 \alpha$ ) levels from mice treated as in L. Representative images shown are from the same samples blotted on different membranes on separate occasions. (N) Liver Nupr1 mRNA levels from mice treated as in $\mathbf{L}$. Data are presented as the mean \pm SEM. ${ }^{*} P<0.05$ for differences between diets/AA level. ${ }^{~} P<0.05$ for effect of $A A$ administration. Statistical tests used were unpaired $t$ test (A and $\mathbf{C}-\mathbf{F})$, 2-way ANOVA with Holm-Sidak post-hoc test (I, J, and $\mathbf{L}-\mathbf{N})$, or 1-way ANOVA with Holm-Sidak post-hoc test (G, H, K). See also Supplemental Figure 7.

$6 \mathrm{H})$. In addition, the higher liver Fgf21 mRNA (Figure 6H) and serum FGF21 (Figure 6I) levels with DPD were partially blunted by NUPR1 knockdown. In line with our prior findings (Figure 5), the metabolic readouts of DPD including feed inefficiency (Figure 6J and Supplemental Figure 6, H and I) and heightened glucose metabolism (Figure 6K and Supplemental Figure 6, J and K) were also partially blunted by liver-specific NUPR1 loss of function.

Meal-related NEAA restriction triggers FGF21 production by hepatocytes. As whole protein is a complex nutrient that can be digested and absorbed as both short peptides and AAs (33), it is possible that either could confer the effects of DPD on FGF21 and subsequent improvements in metabolic control. To investigate the latter, we conducted portal blood AA profiling during DPD refeeding and could demonstrate that nearly all AAs were lower versus $C D$ refeeding (Figure 7A). Moreover, within the liver, we found that Fgf 21 transcripts were localized to hepatocytes surrounding the portal space in DPD-refed mice, as suggested by exclusion of signal from central vein regions (Figure 7B; Fgf21 transcripts were virtually undetectable in samples from mice refed the $\mathrm{CD}$ ). Given this anatomical correlation, we next decided to investigate whether consumption of a diet consisting of protein in the form of free AAs was sufficient to drive the phenotypic responses to DPD. As such, we utilized synthetic AA-containing diets (Supplemental Table 2) mimicking the AA supply that would come from casein, the source of protein in the diets used in our previous experiments (Figures 1-6). In particular, we fed $\mathrm{C} 57 \mathrm{Bl} / 6 \mathrm{~N}$ and NZO mice these diets as was done before (Figures 1 and 3). Dietary AA dilution (DAAD) in lean and obese mice promoted similar changes in body mass accrual and body composition, as was observed with DPD (data not shown). Moreover, DAAD resulted in enhanced insulin sensitivity (Figure 7, C and D) as well as markedly elevated $F g f 21$ transcripts in livers from lean (Figure 7E) and NZO (Figure 7F) mice. Hence, dilution of dietary protein, also when consumed in the form of free AAs, is sufficient to augment hepatic $F g f 21$ and promote metabolic health.

In a cell-autonomous manner, total AA dilution in the culture media also induced $F g f 21$ transcription and the secreted form of the protein in primary mouse hepatocytes (Figure 7, G and H) as well as in clonal Fao rat hepatocytes (Supplemental Figure 7, A and B). As we could observe that both essential AAs (EAAs) and NEAAs were lower in portal blood of DPD-refed mice (Figure 7A), conceivably both could contribute to the induction of $F g f 21$, and so we then tested their requirement in vitro. Surprisingly, Fgf21 and Nurp1 gene expression varied, in an inverse manner, specifically with NEAA concentration in Fao cells (Supplemental Figure 7, C and D). By contrast, enrichment or dilution of only EAAs did not influence transcript levels in Fao cells. Similarly, levels of secreted FGF21 (Figure 7I) and Nupr1 transcripts (Figure 7J) were higher in primary mouse hepatocytes when NEAAs, but not EAAs, were selectively restricted. When NEAAs were added back to AA-diluted media, whether in the indicated pairs (Supplemental Figure $7 E$ ) or individually (data not shown), only the 5 NEAAs glutamate, aspartate, glutamine, asparagine and/or alanine were sufficient to attenuate or prevent the induction of Fgf21 mRNA in Fao cells. Similarly, levels of secreted FGF21 were lower in primary mouse hepatocytes when these 5 NEAAs were added to the background of AA dilution (Figure 7K), indicating that these NEAAs are necessary for the hepatocyte FGF21 response to total AA dilution. Furthermore, single or combined restriction of glutamate/glutamine was sufficient to match the $F g f 21$ induction observed with total AA restriction (Supplemental Figure 7F), indicating that selective dilution of these AAs is sufficient to induce FGF21. Lastly, similar to these in vitro results, we conducted a mouse refeeding study and could demonstrate that administration of the NEAAs Ala, Asn, and Gln, but not the EAAs Phe, Met, and Leu, could retard the DPD-driven increase in serum FGF21 (Figure 7L), liver p-S51eIF2 $\alpha$ (Figure 7M), as well as liver Fgf21 (Supplemental Figure 7G) and $N u p r 1$ (Figure $7 \mathrm{~N}$ ) mRNA in vivo.

Short-term DPD promotes heightened FGF21 and glucose homeostasis reflecting improved metabolic health in young men. Lastly, as others have demonstrated that protein dilution in humans can increase blood FGF21 (16), we wanted to test whether DPD could be beneficial for human health. As such, in a repeated-measures design, after a presampling period, we fed men a diet containing diluted amounts of protein for 7 days, followed by a 7-day normal diet washout period (Supplemental Table 3) without any changes in body mass (data not shown). Dilution of dietary protein had the expected effect of reducing serum urea (Supplemental Figure 8A). Similar to the mouse studies, there was increased serum FGF21 $(\sim 6$-fold; Figure $8 \mathrm{~A})$. Intriguingly, despite no significant change in plasma glucose (Supplemental Figure 8B), fasting plasma insulin levels were decreased (Supplemental Figure 8C) and thus system- 
A
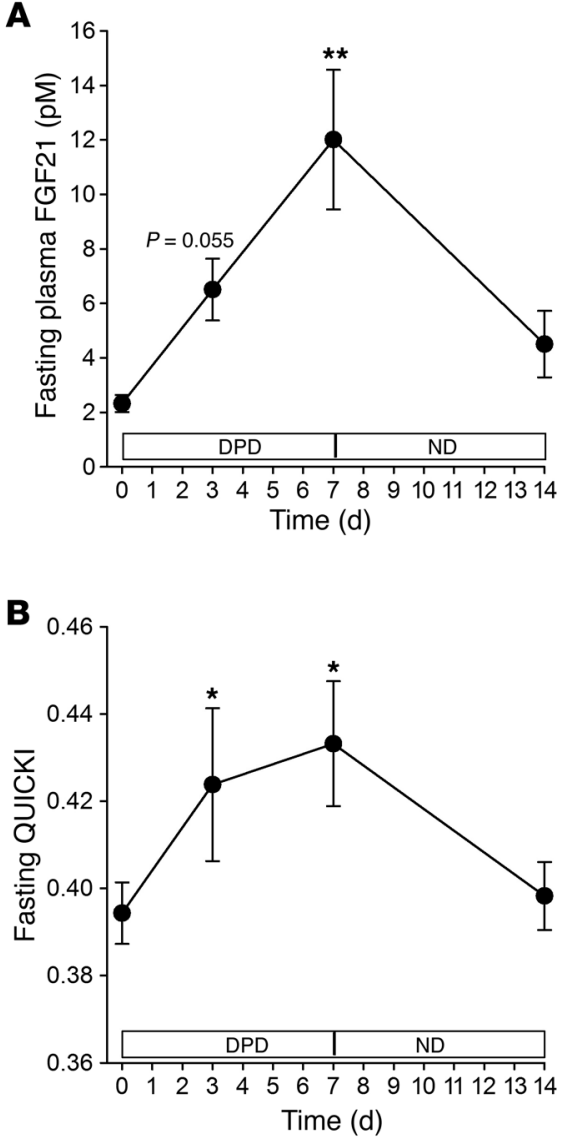

C

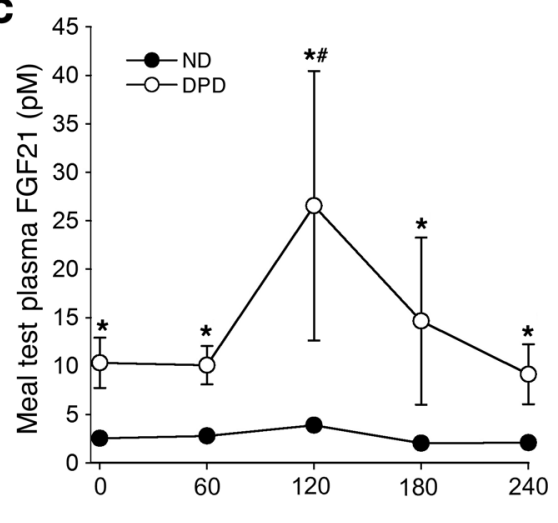

D

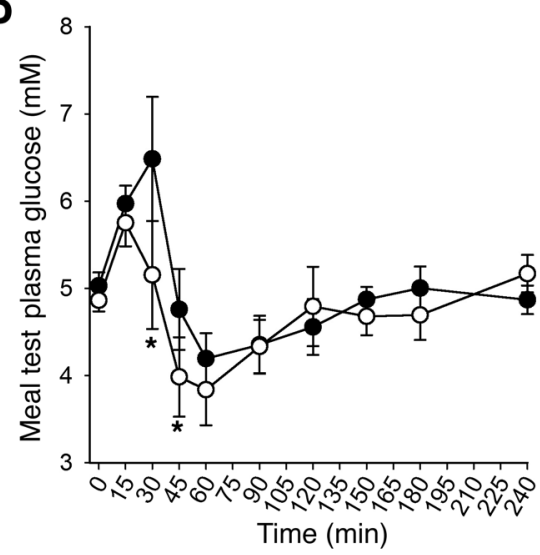

$\mathbf{E}$

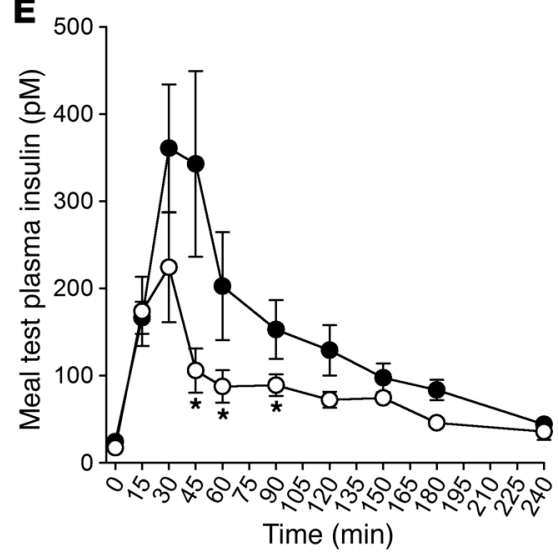

$\mathbf{F}$

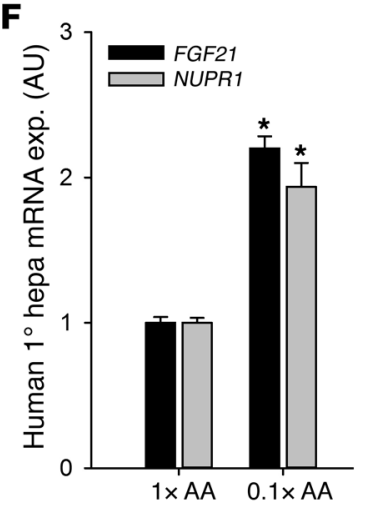

Figure 8. Dietary protein dilution promotes heightened FGF21 and glucose homeostasis, reflecting improved metabolic health in young men. (A) Fasting blood plasma levels of FGF21 in young men subjected to a 7-day period of consumption of a protein-diluted diet (DPD) followed by consumption of their regular mixed diet (ND) for an additional 7 days. $n=5$. (B) Fasting blood plasma quantitative insulin sensitivity check index (QUICKI) in men from $\mathbf{A}$. (C) Blood plasma levels of FGF21 during an equicaloric meal test before (d0) and during (d7) intake of the protein-diluted diet. (D) Blood plasma glucose as in C. (E) Blood plasma insulin as in C. (F) FGF21 and NUPR1 mRNA levels in human primary hepatocytes (Hepas) in response to different media levels of total AA. $n=4$ /condition. Data are the mean \pm SEM. ${ }^{*} P<0.05$ for significant effect of dietary protein/AA level. ${ }^{\#} P<0.05$ for significant effect of time. Statistical tests used were one-way repeated measures ANOVA with Holm-Sidak post-hoc test (A and B), 2-way repeated measures ANOVA with HolmSidak post-hoc test (C-E), and unpaired $t$ test (F). See also Supplemental Figure 8.

ic insulin action was increased (Figure 8B), reflecting an improved glucose homeostasis. Direct testing of this during a meal tolerance test revealed that DPD triggered elevated serum FGF21 levels (Figure 8C) and, despite consuming more carbohydrate, lower circulating glucose (Figure 8D) and insulin (Figure 8E). There were no differences in fasting levels of plasma NEFA (Supplemental Figure 8D) or triglycerides (Supplemental Figure 8E). There was also lower serum IGF1 ( $40 \%$; Supplemental Figure 8F) levels with DPD, and serum adiponectin remained unchanged (Supplemental Figure 8G). Lastly, similarly to our mouse studies, media AA dilution also elevated FGF21 and NUPR1 mRNA levels in human primary hepatocytes (Figure 8F).

\section{Discussion}

Here we show that DPD promotes metabolic health in humans and mice. In mouse models of obesity/T2D, DPD reduced metabolic efficiency, despite higher food energy intake and adiposity. While the effects of DPD did not require adipose UCP1 expression, DPD-induced liver FGF21 was required for the improved glucose metabolism and metabolic inefficiency with DPD, both in lean and obese mice. An induction of liver NUPR1 was required, at least in part, for increased FGF21 and associated metabolic remodeling with DPD. Furthermore, an insufficiency of NEAAs, particularly alanine, glutamine/glutamate, and asparagine/aspartate was necessary and sufficient for hepatocyte NUPR1-driven FGF21 induction and secretion.

We show that liver-derived serum FGF21 is required for the metabolic remodeling that follows DPD. In support of this conclusion, heightened FGF21 levels have been observed previously with DPD (34), with a requirement of systemic FGF21 for the promotion of metabolic inefficiency with DPD (16). In particular, we found that circulating FGF21 levels seem to be markedly induced in the fed state, within hours following ingestion of a protein-diluted meal. Interestingly, fructose or glucose ingestion rapidly induced FGF21 in humans (35), suggesting that FGF21 levels may in fact be highly responsive to nutrient balance following meal ingestion. Nevertheless, despite the induction by DPD being roughly similar, serum FGF21 levels were markedly lower in humans compared with mice. Thus, even though pharmacological FGF21 administration in humans results in similar responses to those seen in mice (36), further studies are warranted 
to determine whether the increase in FGF21 is related to improved glucose metabolism with DPD in humans.

Conceivably, the improved glucose homeostasis with DPD could result from a correction in dysregulated endogenous glucose production and/or systemic glucose clearance (37). Indeed, select AAs are key gluconeogenic substrates in the liver (38), and in the present study DPD blunted endogenous glucose production as assessed by pyruvate tolerance testing. However, whether AAs substantially contribute to dysregulated hepatic glucose production in T2D is presently unclear, and given that DPD mice had heightened systemic FA oxidation, a potential lower systemic FA and/or glycerol supply to the liver $(39,40)$ could lead to a correction of excessive hepatic glucose production in obese DPD mice. On the other hand, DPD consistently increased glucose uptake in BAT and the heart, which are major sites of impaired glucose clearance in obesity/T2D (41), indicating that heightened glucose clearance by these tissues contributes to the improved glucose homeostasis. Importantly, we have previously demonstrated that neutral AA restriction in mice by germline deletion of an intestinal and renal AA transporter results in improved glucose homeostasis in obesity and enhanced glucose clearance by the heart (42). The molecular physiological mechanisms by which reduced systemic AA supply enhances systemic glucose clearance are presently vague but certainly involve FGF21. Indeed, FGF21 can increase systemic glucose disposal either via direct effects as well as indirectly via acting through the central nervous system-driven sympathetic nervous activity (43).

Regarding dietary protein, dietary AAs were sufficient to mimic the effects of DPD to increase FGF21 and metabolic inefficiency, which is consistent with studies that show similar (42) or disparate (33) phenotypes with deficient intestinal neutral AA or peptide transport capacity, respectively. The connection between dietary AA supply and metabolism has been largely focused upon restriction or deprivation of the EAAs. For example, single EAA restriction $(44,45)$ or deprivation $(46,47)$ has been associated with higher levels of circulating FGF21, alongside elevated energy expenditure and insulin sensitivity. Evidence is emerging, however, that hepatic FGF21 can also be regulated by NEAA supply. For instance, mice with relative hepatic asparagine/glutamine $(48,49)$ or alanine $(50)$ insufficiency also have higher hepatic and circulating FGF21 levels. Consistent with these observations, our data provide evidence that hepatocyte FGF21 induction following DPD occurs in response to an insufficiency of NEAAs, specifically Ala, Gln/Glu, and Asn/Asp. It will be interesting to further determine the biochemical bases by which the deprivation of select NEAAs affects FGF21 expression.

Concerning the molecular bases by which DPD affects liver FGF21 expression/secretion, we reveal here that NUPR1 expression in the liver/hepatocyte is induced by DPD/AAD in vivo and in vitro and that it is required, at least in part, for the induction of Fgf21, and associated metabolic remodeling, therein. NUPR1 is known as a multifunctional stress-induced protein and regulates diverse responses depending on cell and stress type (30). NUPR1 contains a basic helix-loop-helix motif reminiscent of certain transcription factors, and experimental evidence supports a role for NUPR1 in transcriptional control (51). As NUPR1 has previously been identified to have a role in the ISR pathway (24) and the ISR is implicated in induction of liver FGF21 by dietary protein or AA deprivation (Figure 6 and refs. 16, 46, and 52), NUPR1 induction thus represents a potentially novel molecular component driving the liver FGF21 induction in response to DPD, perhaps as a feedforward reinforcer of the ISR (23).

Similarly to prior studies in rats (53) and mice (16), we could consistently show that DPD promotes metabolic inefficiency, owing to heightened energy expenditure (EE). Earlier studies by Stock and colleagues showed that DPD could promote EE and heat generation and coined this as diet-induced thermogenesis (54). Although the metabolic origins of heightened EE with DPD are likely to be diverse, there is strong evidence that this involves BAT (55), perhaps via UCP1. Although UCP1 is considered one principal mediator of metabolic inefficiency by means of mitochondrial uncoupling (20), the DPD-induced metabolic remodeling was exacerbated in UCP1 knockout mice. Therefore, DPDinduced metabolic inefficiency is independent of mitochondrial uncoupling involving UCP1 and thus other adaptive mechanisms, probably involving FGF21 (Figures 4-6) and perhaps leptin signaling (56), are likely to be involved. In any case, even though the improved glucose homeostasis consistently coincided with metabolic inefficiency with DPD, whether enhanced EE causes improved metabolic health with DPD is presently unclear, as others have observed that these phenomena can be disconnected (57).

In obese/T2D mouse models, although heightened FGF21 was required for the effects of DPD to improve glucose metabolism, we observed a disconnection between obesity and metabolic health and there was not a strict relationship between serum FGF21 and glucose homeostasis/metabolic efficiency, indicating the presence of other homeostatic mechanisms. For instance, the weight gain despite protein dilution in the high-fat dietary condition could be mediated by a lack of an inhibitory signal on food intake generated from low carbohydrate intake, ultimately relating to the dietary protein to carbohydrate ratio (11). Weight lossindependent improvements in glucose metabolism have been observed in other mouse models $(58,59)$, and have also been observed with common therapeutic interventions such as insulin and thiazolidinedione, suggesting that factors other than obesity per se lead to metabolic dysfunction. Along with body fat distribution, improved metabolic homeostasis most likely relates to the manner in which adipocytes within adipose tissue coordinate lipid metabolism and interact with other cell types such that an attenuation of inflammation and fibrosis allows a healthy expansion of the adipose organ(s) (60). Indeed, regardless of the direction, we could consistently show FGF21-dependent WAT plasticity upon DPD. Thus, it is tempting to speculate that an environmental variable such as DPD may explain the subset of human populations displaying insulin-sensitive obesity (61).

Lastly, it is known that DPD can extend the lifespan of rodents $(11,62)$ and retard aging-related illnesses such as certain cancers (63), Alzheimer's disease (64), and T2D (Figure 4). While the precise mechanisms involved are not clear, they may include, but are not limited to, improved stress resistance (65), lowered insulin/ IGF1 levels (66), heightened FGF21 (67), or enhanced metabolic rate (68) with DPD.

In summary, dietary dilution of protein/AAs promotes improved metabolic health in mice and men, at least in part through a select NEAA insufficiency-induced liver NUPR1-FGF21 axis. 


\section{Methods}

Full experimental details can be found within the Supplemental Methods.

Animal experiments. Mice used were male C57Bl/6NCrl (cata$\log$ 027, Charles River Laboratories), as well as New Zealand Black (catalog 000993, NZB/BlNJ) and New Zealand Obese (catalog 002105, NZO/HlLtJ, Jackson Laboratories) strains. In addition,

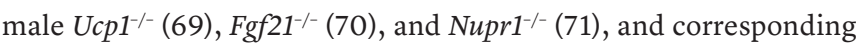
+ + + littermate mice from +/- crossings on the C57Bl/6J background were used. For liver-specific gene expression manipulations, mice were tail-vein administered Fgf21 shR, Nupr1 shR, or control shR adenoviruses. For ISRIB experiments, mice were intraperitoneally administered trans-ISRIB (catalog 5095840001; Merck-Millipore; $2.5 \mathrm{mg} / \mathrm{kg} ; \sim 60 \mu \mathrm{g}$ in $100 \mu \mathrm{l}$ solution composed of $12.5 \%$ DMSO, $12.5 \%$ PEG400, $75 \% 0.9 \% \mathrm{NaCl}$ ) or corresponding vehicle (28). Details of the diet (Research Diets Inc.) compositions are outlined in Supplemental Tables 1 and 2.

Behavioral and metabolic phenotyping. For all metabolic experiments, general considerations outlined by the EMPRESS protocols (http://empress.har.mrc.ac.uk) were followed. Simultaneous determination of indirect calorimetry, 3D activity, and food consumption was conducted using the PhenoMaster Cage System (TSE Systems). Twenty-four-hour urine collection was performed in custom-made individual housing units as conducted previously (72). Nutrient oxidation rates were calculated from 24-hour rates of $\mathrm{O}_{2}$ consumption, $\mathrm{CO}_{2}$ production, and urinary urea production as described (19). Feed efficiency was calculated based upon the quotient of body mass accrual (mg) and food energy intake (kJ) during a selected growth period.

Assessment of glucose homeostasis. Blood glucose measurements for all tests were determined with an Accu-Chek Performa glucometer (Roche). Glucose, insulin, and pyruvate tolerance tests were conducted according to established guidelines (73). For the assessment of tissue glucose uptake in vivo, we used methods essentially as described previously (74). A fasting ISI was calculated using the equation ISI $=1,000 /($ blood glucose $[\mathrm{mM}] \times$ serum insulin $[\mathrm{pM}])$ from 5- to 6-hour-fasted mice.

Human experiments. Five healthy, lean, male volunteers, age $25.6 \pm$ 0.4 years, body weight $75.9 \pm 5.3 \mathrm{~kg}$ (mean \pm SEM), participated. Subjects consumed a controlled diet low in protein ( $~ 9 \%$ total caloric energy from protein; $12.8 \mathrm{MJ} / \mathrm{d}$ ) for 7 days followed by a washout period for 7 days on their habitual, mixed diet ( $20 \%$ total caloric energy from protein; $\sim 9.4 \mathrm{MJ} / \mathrm{d}$ ) and protein intake averaged $0.94 \pm 0.03$ and $1.57 \pm$ $0.22 \mathrm{~g} / \mathrm{kg} / \mathrm{d}$ in the DPD and habitual diet, respectively (Supplemental Table 3). Blood samples were obtained at selected time points in the morning in the postabsorptive state.

Cell culture. Fao rat hepatoma cells (75) (catalog 89042701, SigmaAldrich), primary mouse hepatocytes (PMHs) (76), and primary human hepatocytes (PHHs; HMCPIS, lot HU1359, Life Technologies) were used and plated according to standard techniques. To conduct AA restriction studies, custom media were made from $10 \times$ HBSS (Fao) or 2× William's Media E (PMHs and PHHs; Genaxxon Bioscience $\mathrm{GmbH}$ ). AAs at RPMI (Fao) or mimicking rat portal vein (PHMs and PHHs) concentrations were added as indicated.

Serum, urine, media metabolite, and hormone analyses. For mouse samples, commercially available kits were used to measure serum and urine metabolites and hormones. Serum AA profiling was conducted using liquid chromatography-tandem mass spectrometry as described previously in detail (42).
RNA/protein extraction and analysis. RNA was extracted from tissues using QIAzol and cDNA was synthesized using the Quantitect Reverse Transcription Kit (QIAGEN). qPCR was conducted using Taqman master mix and Taqman primer-probe assays (Life Technologies). For comparison of spliced (mature mRNA) versus unspliced (pre-mRNA) Fgf 21 transcripts, amplicons spanning exons 2 to 3 (for mature mRNA) or exon $1+$ intron 1 (for pre-mRNA) were quantified using Quantitect Sybr Green qPCR (QIAGEN) with the following primers: Mature Fwd, CAGGGGTCATTCAAATCCTG; Mature Rev, CTGGTTTGGGGAGTCCTTCT; pre-mRNA Fwd, CTGGGGGTCTACCAAGCATA; pre-mRNA Rev AGCCTCCTCCTCTCAACCTC. Tissue protein extraction and immunoblotting were performed using standard methods with antibodies against UCP1 (catalog 662045, Calbiochem), p-S51-eIF2 $\alpha$ (catalog 9721, Cell Signaling Technology), NUPR1 (catalog SAB1104559, Sigma-Aldrich), and the housekeeping protein HSP90 (catalog 610418, BD Biosciences).

Statistics. For 1-factorial designs, 2-tailed unpaired $t$ tests were performed. For multiple comparisons, statistical analyses were performed using a 1-way or 2-way ANOVA, with or without repeated measures, with Holm-Sidak post-hoc tests when significant differences were detected, where appropriate (Microsoft Excel and SigmaPlot 13 [Systat Software $\mathrm{GmbH}$ ]). The significance level was set at $P$ less than 0.05 .

Study approval. Animal experiments were conducted according to local, national, and EU ethical guidelines (Regierungspräsidium Karlsruhe), and adhered to ARRIVE guidelines. For the human study, all subjects gave informed consent prior to their participation and the study was approved by the Copenhagen Ethics Committee (H-3-2012129) and was executed in accordance with the code of ethics of the World Medical Association (Helsinki II declaration).

\section{Author contributions}

Designed and conducted experiments: AM, AZ, KAS, JS, TPS, AP, MMC, TG, JF, UR, BK, and AJR. Analyzed data: AM, KAS, JS, TPS, BK, and AJR. Provided materials, infrastructure and support: DS, $\mathrm{MH}$, JLI, BK, SH, and AJR. Wrote the manuscript: AM and AJR. Edited the manuscript: AM, DS, BK, SH, and AJR.

\section{Acknowledgments}

The authors acknowledge the experimental support from Oksana Seibert, Yvonne Feuchter, Jessica Chan, Joshua Mouatt, Anja Reimann, Maximilian Schuster, and Hermann-Josef Gröne (DKFZ). We thank Martin Klingenspor (TUM, Germany) for the provision of the UCP1 knockout mice breeding pairs and Emerald Perlas (EMBL) for experimental support for ISH assays. AM was supported by a CIHR fellowship. The human diet study was supported by the grant "Physical Activity and Nutrition for Improvement of Health" from the University of Copenhagen Excellence Program for Interdisciplinary Research (2016) to BK. These studies were supported by the Helmholtz Programs "ICEMED” and "Metabolic Dysfunction" as well as the Deutsche Forschungsgemeinschaft (He3260/8-1) to SH and by a project grant from the EFSD/Lilly European Diabetes Research Programme to AJR.

Address correspondence to: Adam J. Rose, DKFZ-A170, Im Neuenheimer Feld 280, Heidelberg, Germany, 69210. Phone: 49.6221.423588; E-mail: a.rose@dkfz.de. 
1. Popkin BM, Adair LS, Ng SW. Global nutrition transition and the pandemic of obesity in developing countries. Nutr Rev. 2012;70(1):3-21.

2. Ravussin E, Bogardus C. Energy balance and weight regulation: genetics versus environment. Br J Nutr. 2000;83 Suppl 1:S17-S20.

3. Schutz Y. Macronutrients and energy balance in obesity. Metab Clin Exp. 1995;44(9 Suppl 3):7-11.

4. Keller U. Dietary proteins in obesity and in diabetes. Int J Vitam Nutr Res. 2011;81(2-3):125-133.

5. Franz MJ. Protein and diabetes: much advice, little research. Curr Diab Rep. 2002;2(5):457-464.

6. Morrison CD, Reed SD, Henagan TM. Homeostatic regulation of protein intake: in search of a mechanism. Am J Physiol Regul Integr Comp Physiol. 2012;302(8):R917-R928.

7. Du F, Higginbotham DA, White BD. Food intake, energy balance and serum leptin concentrations in rats fed low-protein diets. J Nutr. 2000;130(3):514-521.

8. Sørensen A, Mayntz D, Raubenheimer D, Simpson SJ. Protein-leverage in mice: the geometry of macronutrient balancing and consequences for fat deposition. Obesity (Silver Spring). 2008;16(3):566-571.

9. Gosby AK, et al. Testing protein leverage in lean humans: a randomised controlled experimental study. PLoS One. 2011;6(10):e25929.

10. Simpson SJ, Raubenheimer D. Obesity: the protein leverage hypothesis. Obes Rev. 2005;6(2):133-142.

11. Solon-Biet SM, et al. The ratio of macronutrients, not caloric intake, dictates cardiometabolic health, aging, and longevity in ad libitum-fed mice. Cell Metab. 2014;19(3):418-430.

12. Rossetti L, Rothman DL, DeFronzo RA, Shulman GI. Effect of dietary protein on in vivo insulin action and liver glycogen repletion. Am J Physiol. 1989;257(2 Pt 1):E212-E219.

13. Newgard CB, et al. A branched-chain amino acidrelated metabolic signature that differentiates obese and lean humans and contributes to insulin resistance. Cell Metab. 2009;9(4):311-326.

14. Krebs M, et al. Mechanism of amino acidinduced skeletal muscle insulin resistance in humans. Diabetes. 2002;51(3):599-605.

15. van Nielen $M$, et al. Dietary protein intake and incidence of type 2 diabetes in Europe: the EPICInterAct Case-Cohort Study. Diabetes Care. 2014;37(7):1854-1862.

16. Laeger T, et al. FGF21 is an endocrine signal of protein restriction. JClin Invest. 2014;124(9):3913-3922.

17. Tseng YH, Cypess AM, Kahn CR. Cellular bioenergetics as a target for obesity therapy. Nat Rev Drug Discov. 2010;9(6):465-482.

18. Stock MJ. Gluttony and thermogenesis revisited. Int J Obes Relat Metab Disord. 1999;23(11):1105-1117.

19. Frayn KN. Calculation of substrate oxidation rates in vivo from gaseous exchange. J Appl Physiol Respir Environ Exerc Physiol. 1983;55(2):628-634.

20. Nedergaard J, Golozoubova V, Matthias A, Asadi A, Jacobsson A, Cannon B. UCP1: the only protein able to mediate adaptive non-shivering thermogenesis and metabolic inefficiency. Biochim Biophys Acta. 2001;1504(1):82-106.

21. Cannon B, Nedergaard J. Nonshivering thermo- genesis and its adequate measurement in metabolic studies. J Exp Biol. 2011;214(Pt 2):242-253.

22. Baird TD, Wek RC. Eukaryotic initiation factor 2 phosphorylation and translational control in metabolism. Adv Nutr. 2012;3(3):307-321.

23. Jin HO, et al. Nuclear protein 1 induced by ATF 4 in response to various stressors acts as a positive regulator on the transcriptional activation of ATF4. IUBMB Life. 2009;61(12):1153-1158.

24. Averous J, et al. Amino acid deprivation regulates the stress-inducible gene $\mathrm{p} 8$ via the GCN2/ ATF4 pathway. Biochem Biophys Res Commun. 2011;413(1):24-29.

25. Shan J, Lopez MC, Baker HV, Kilberg MS. Expression profiling after activation of amino acid deprivation response in HepG2 human hepatoma cells. Physiol Genomics. 2010;41(3):315-327.

26. Jousse $\mathrm{C}$, et al. TRB3 inhibits the transcriptional activation of stress-regulated genes by a negative feedback on the ATF 4 pathway. J Biol Chem. 2007;282(21):15851-15861.

27. Ye J, et al. GCN2 sustains mTORC1 suppression upon amino acid deprivation by inducing Sestrin2. Genes Dev. 2015;29(22):2331-2336.

28. Sidrauski C, et al. Pharmacological brake-release of mRNA translation enhances cognitive memory. Elife. 2013;2:e00498.

29. Sidrauski C, McGeachy AM, Ingolia NT, Walter P. The small molecule ISRIB reverses the effects of eIF $2 \alpha$ phosphorylation on translation and stress granule assembly. Elife. 2015;4:e05033.

30. Goruppi S, Iovanna JL. Stress-inducible protein p8 is involved in several physiological and pathological processes. J Biol Chem. 2010;285(3):1577-1581.

31. Barbosa-Sampaio HC, et al. Nupr1 deletion protects against glucose intolerance by increasing beta cell mass. Diabetologia. 2013;56(11):2477-2486.

32. Kong DK, et al. Deficiency of the transcriptional regulator $\mathrm{p} 8$ results in increased autophagy and apoptosis, and causes impaired heart function. Mol Biol Cell. 2010;21(8):1335-1349.

33. Nässl AM, Rubio-Aliaga I, Fenselau H, Marth MK, Kottra G, Daniel H. Amino acid absorption and homeostasis in mice lacking the intestinal peptide transporter PEPT1. Am J Physiol Gastrointest Liver Physiol. 2011;301(1):G128-G137.

34. Bielohuby M, et al. Impaired glucose tolerance in rats fed low-carbohydrate, highfat diets. Am J Physiol Endocrinol Metab. 2013;305(9):E1059-E1070.

35. Dushay JR, Toschi E, Mitten EK, Fisher FM, Herman MA, Maratos-Flier E. Fructose ingestion acutely stimulates circulating FGF21 levels in humans. Mol Metab. 2015;4(1):51-57.

36. Gimeno RE, Moller DE. FGF21-based pharmacotherapy--potential utility for metabolic disorders. Trends Endocrinol Metab. 2014;25(6):303-311.

37. Gerich JE. Physiology of glucose homeostasis. Diabetes Obes Metab. 2000;2(6):345-350.

38. Jungas RL, Halperin ML, Brosnan JT. Quantitative analysis of amino acid oxidation and related gluconeogenesis in humans. Physiol Rev. 1992;72(2):419-448.

39. Perry RJ, et al. Hepatic acetyl CoA links adipose tissue inflammation to hepatic insulin resistance and type 2 diabetes. Cell. 2015;160(4):745-758.

40. Mittelman SD, Fu YY, Rebrin K, Steil G, Bergman RN. Indirect effect of insulin to suppress endogenous glucose production is dominant, even with hyperglucagonemia. JClin Invest. 1997;100(12):3121-3130.

41. Cooney GJ, Astbury LD, Williams PF, Caterson ID. Insulin response in individual tissues of control and gold thioglucose-obese mice in vivo with $\left[1{ }^{-14} \mathrm{C}\right] 2$-deoxyglucose. Diabetes. 1987;36(2):152-158.

42. Jiang $Y$, et al. Mice lacking neutral amino acid transporter B(0)AT1 (Slc6a19) have elevated levels of FGF21 and GLP-1 and improved glycaemic control. Mol Metab. 2015;4(5):406-417.

43. Owen BM, Mangelsdorf DJ, Kliewer SA. Tissuespecific actions of the metabolic hormones FGF15/19 and FGF21. Trends Endocrinol Metab. 2015;26(1):22-29.

44. Lees EK, et al. Methionine restriction restores a younger metabolic phenotype in adult mice with alterations in fibroblast growth factor 21. Aging Cell. 2014;13(5):817-827.

45. Stone KP, Wanders D, Orgeron M, Cortez CC, Gettys TW. Mechanisms of increased in vivo insulin sensitivity by dietary methionine restriction in mice. Diabetes. 2014;63(11):3721-3733.

46. De Sousa-Coelho AL, et al. FGF21 mediates the lipid metabolism response to amino acid starvation. J Lipid Res. 2013;54(7):1786-1797.

47. Fisher FM, et al. Fibroblast growth factor 21 limits lipotoxicity by promoting hepatic fatty acid activation in mice on methionine and choline-deficient diets. Gastroenterology. 2014;147(5):1073-1083.e6.

48. Cornu M, et al. Hepatic mTORC1 controls locomotor activity, body temperature, and lipid metabolism through FGF21. Proc Natl Acad Sci USA. 2014;111(32):11592-11599.

49. Wilson GJ, et al. GCN2 is required to increase fibroblast growth factor 21 and maintain hepatic triglyceride homeostasis during asparaginase treatment. Am J Physiol Endocrinol Metab. 2015;308(4):E283-E293.

50. Shimizu N, et al. A muscle-liver-fat signalling axis is essential for central control of adaptive adipose remodelling. Nat Commun. 2015;6:6693.

51. Cano CE, Hamidi T, Sandi MJ, Iovanna JL. Nupr1: the Swiss-knife of cancer. JCell Physiol. 2011;226(6):1439-1443

52. Wanders D, et al. Role of GCN2-independent signaling through a noncanonical PERK/ NRF2 pathway in the physiological responses to dietary methionine restriction. Diabetes. 2016;65(6):1499-1510.

53. Rothwell NJ, Stock MJ, Tyzbir RS. Energy balance and mitochondrial function in liver and brown fat of rats fed "cafeteria" diets of varying protein content. J Nutr. 1982;112(9):1663-1672.

54. Rothwell NJ, Stock MJ. A role for brown adipose tissue in diet-induced thermogenesis. Nature. 1979;281(5726):31-35.

55. Stock MJ, Rothwell NJ. The role of brown fat in diet-induced thermogenesis. Int J Vitam Nutr Res. 1986;56(2):205-210.

56. Specter SE, Hamilton JS, Stern JS, Horwitz BA. Chronic protein restriction does not alter energetic efficiency or brown adipose tissue thermogenic capacity in genetically obese (fa/fa) Zucker rats. J Nutr. 1995;125(8):2183-2193.

57. Wanders $\mathrm{D}$, et al. UCP1 is an essential mediator 
of the effects of methionine restriction on energy balance but not insulin sensitivity. FASEB J. 2015;29(6):2603-2615.

58. Kim JY, et al. Obesity-associated improvements in metabolic profile through expansion of adipose tissue. J Clin Invest. 2007;117(9):2621-2637.

59. Renne U, et al. Lifelong obesity in a polygenic mouse model prevents age- and dietinduced glucose intolerance- obesity is no road to late-onset diabetes in mice. PLoS One. 2013;8(11):e79788.

60. Sun K, Tordjman J, Clément K, Scherer PE. Fibrosis and adipose tissue dysfunction. Cell Metab. 2013;18(4):470-477.

61. Blüher M. The distinction of metabolically 'healthy' from 'unhealthy' obese individuals. Curr Opin Lipidol. 2010;21(1):38-43.

62. Miller DS, Payne PR. Longevity and protein intake. Exp Gerontol. 1968;3(3):231-234.

63. Levine ME, et al. Low protein intake is associated with a major reduction in IGF-1, cancer, and overall mortality in the 65 and younger but not older population. Cell Metab. 2014;19(3):407-417. 64. Parrella E, et al. Protein restriction cycles reduce
IGF-1 and phosphorylated Tau, and improve behavioral performance in an Alzheimer's disease mouse model. Aging Cell. 2013;12(2):257-268.

65. Harputlugil E, Hine C, Vargas D, Robertson L, Manning BD, Mitchell JR. The TSC complex is required for the benefits of dietary protein restriction on stress resistance in vivo. Cell Rep. 2014;8(4):1160-1170.

66. Sadagurski M, White MF. Integrating metabolism and longevity through insulin and IGF1 signaling. Endocrinol Metab Clin North Am. 2013;42(1):127-148.

67. Zhang Y, et al. The starvation hormone, fibroblast growth factor-21, extends lifespan in mice. Elife. 2012;1:e00065.

68. Speakman JR. Body size, energy metabolism and lifespan. J Exp Biol. 2005;208(Pt 9):1717-1730.

69. Enerbäck S, et al. Mice lacking mitochondrial uncoupling protein are cold-sensitive but not obese. Nature. 1997;387(6628):90-94.

70. Hotta Y, et al. Fibroblast growth factor 21 regulates lipolysis in white adipose tissue but is not required for ketogenesis and triglyceride clearance in liver. Endocrinology. 2009;150(10):4625-4633.
71. Vasseur S, et al.p8-deficient fibroblasts grow more rapidly and are more resistant to adriamycin-induced apoptosis. Oncogene. 2002;21(11):1685-1694.

72. Stettner $P$, et al. Sulfatides are required for renal adaptation to chronic metabolic acidosis. Proc Natl Acad Sci USA. 2013;110(24):9998-10003.

73. Ayala JE, et al. Standard operating procedures for describing and performing metabolic tests of glucose homeostasis in mice. Dis Model Mech. 2010;3(9-10):525-534.

74. Maarbjerg SJ, et al. Genetic impairment of AMPKalpha2 signaling does not reduce muscle glucose uptake during treadmill exercise in mice. Am J Physiol Endocrinol Metab. 2009;297(4):E924-E934.

75. Deschatrette J, Weiss MC. Characterization of differentiated and dedifferentiated clones from a rat hepatoma. Biochimie. 1974;56(11-12):1603-1611.

76. Klingmüller U, et al. Primary mouse hepatocytes for systems biology approaches: a standardized in vitro system for modelling of signal transduction pathways. Syst Biol (Stevenage). 2006;153(6):433-447. 